\title{
Synthesis and Characterization of Trimetallic Ruthenium and Bimetallic Osmium Complexes with Metal-vinyl Linkages
}

Haiping Xia, ${ }^{\mathrm{a}}$ Ting Bin Wen, ${ }^{\mathrm{a}}$ Quan Yuan $\mathrm{Hu},{ }^{\mathrm{a}}$ Xin Wang, ${ }^{\mathrm{a}}$ Xingguo Chen, ${ }^{\mathrm{a}}$ Lai Yung Shek, ${ }^{a}$ Ian D. Williams, ${ }^{a}$ Kam Sing Wong, ${ }^{\mathrm{b}, *}$ George K. L. Wong ${ }^{\mathrm{b}, *}$ and Guochen Jia ${ }^{\mathrm{a}, *}$ aDepartment of Chemistry and Open Laboratory of Chirotechnology of the Institute of Molecular Technology for Drug Discovery and Synthesis and bepartment of Physics, The Hong Kong University of Science and Technology, Clear Water Bay, Kowloon, Hong Kong

\section{Supporting Information Available}

Table S1. Atomic coordinates ( x 104) and equivalent isotropic displacement parameters $\left(\AA^{2} \mathrm{x} \quad 10^{3}\right)$ for $1,3,5-\left[\mathrm{Cl}(\mathrm{CO})(\mathrm{Py})\left(\mathrm{PPh}_{3}\right)_{2} \mathrm{RuCH}=\mathrm{CH}\right]_{3} \mathrm{C}_{6} \mathrm{H}_{3} \mathrm{CH}_{2} \mathrm{Cl}_{2}$ $\left(5 \cdot \mathrm{CH}_{2} \mathrm{Cl}_{2}\right)$

Table S2. Bond lengths [Å] for 1,3,5-[Cl(CO)(Py) $\left.\left(\mathrm{PPh}_{3}\right)_{2} \mathrm{RuCH}=\mathrm{CH}\right]_{3} \mathrm{C}_{6} \mathrm{H}_{3} \cdot \mathrm{CH}_{2} \mathrm{Cl}_{2}$ $\left(5 \cdot \mathrm{CH}_{2} \mathrm{Cl}_{2}\right)$

Table S3. Bond angles $\left[^{\circ}\right]$ for $1,3,5-\left[\mathrm{Cl}(\mathrm{CO})(\mathrm{Py})\left(\mathrm{PPh}_{3}\right)_{2} \mathrm{RuCH}=\mathrm{CH}\right]_{3} \mathrm{C}_{6} \mathrm{H}_{3} \mathrm{CH}_{2} \mathrm{Cl}_{2}$ $\left(5 \cdot \mathrm{CH}_{2} \mathrm{Cl}_{2}\right)$

Table S4. Anisotropic displacement parameters $\left(\AA^{2} \times 1^{3}\right)$ for $1,3,5-$ $\left[\mathrm{Cl}(\mathrm{CO})(\mathrm{Py})\left(\mathrm{PPh}_{3}\right)_{2} \mathrm{RuCH}=\mathrm{CH}\right]_{3} \mathrm{C}_{6} \mathrm{H}_{3} \cdot \mathrm{CH}_{2} \mathrm{Cl}_{2}\left(\mathbf{5} \cdot \mathrm{CH}_{2} \mathrm{Cl}_{2}\right)$

Table S5. Hydrogen coordinates $\left(\times 10^{4}\right)$ and isotropic displacement parameters $\left(\AA^{2} \times\right.$ $\left.10^{3}\right)$ for $1,3,5-\left[\mathrm{Cl}(\mathrm{CO})(\mathrm{Py})\left(\mathrm{PPh}_{3}\right)_{2} \mathrm{RuCH}=\mathrm{CH}\right]_{3} \mathrm{C}_{6} \mathrm{H}_{3} \cdot \mathrm{CH}_{2} \mathrm{Cl}_{2}\left(\mathbf{5} \cdot \mathrm{CH}_{2} \mathrm{Cl}_{2}\right)$

Table S6. Atomic coordinates $\left(\times 10^{4}\right)$ and equivalent isotropic displacement 
parameters $\left(\AA^{2} \times 10^{3}\right)$ for $\mathrm{OsCl}(\mathrm{CH}=\mathrm{CH}(p$-tolyl $))(\mathrm{CO})\left(\mathrm{PPh}_{3}\right) 3 \cdot 2 \mathrm{CH}_{2} \mathrm{Cl}_{2}\left(\mathbf{8 b} \cdot 2 \mathrm{CH}_{2} \mathrm{Cl}_{2}\right)$

Table S7. Bond lengths $(\AA)$ and angles $\left(^{\circ}\right)$ for $\mathrm{OsCl}(\mathrm{CH}=\mathrm{CH}(p-$

tolyl) $(\mathrm{CO})(\mathrm{PPh} 3) 3 \cdot 2 \mathrm{CH}_{2} \mathrm{Cl}_{2}\left(\mathbf{8 b} \cdot 2 \mathrm{CH}_{2} \mathrm{Cl}_{2}\right)$

Table S8. Anisotropic displacement parameters $\left(\AA^{2} \times 10^{3}\right)$ for $\mathrm{OsCl}(\mathrm{CH}=\mathrm{CH}(p$ tolyl) $(\mathrm{CO})(\mathrm{PPh} 3) 3 \cdot 2 \mathrm{CH}_{2} \mathrm{Cl}_{2}\left(\mathbf{8 b} \cdot 2 \mathrm{CH}_{2} \mathrm{Cl}_{2}\right)$

Table 9. Hydrogen coordinates $\left(\times 10^{4}\right)$ and isotropic displacement parameters $\left(\AA^{2} \mathrm{x}\right.$ $\left.10^{3}\right)$ for $\mathrm{OsCl}(\mathrm{CH}=\mathrm{CH}(p$-tolyl $))(\mathrm{CO})\left(\mathrm{PPh}_{3}\right) 3 \cdot 2 \mathrm{CH}_{2} \mathrm{Cl}_{2}\left(\mathbf{8 b} \cdot 2 \mathrm{CH}_{2} \mathrm{Cl}_{2}\right)$ 
Table S1. Atomic coordinates ( $\mathrm{x} 10^{4}$ ) and equivalent isotropic displacement parameters $\left(\AA^{2} \times 10^{3}\right)$ for $1,3,5-\left[\mathrm{Cl}(\mathrm{CO})(\mathrm{Py})\left(\mathrm{PPh}_{3}\right)_{2} \mathrm{RuCH}=\mathrm{CH}\right]_{3} \mathrm{C}_{6} \mathrm{H}_{3} \cdot \mathrm{CH}_{2} \mathrm{Cl}_{2}\left(\mathbf{5}^{\circ} \mathrm{CH}_{2} \mathrm{Cl}_{2}\right)$.

\begin{tabular}{|c|c|c|c|c|}
\hline Atom & $x$ & $y$ & $z$ & $U(e q)$ \\
\hline $\operatorname{Ru}(1)$ & $7806(1)$ & $5131(1)$ & 951(1) & $50(1)$ \\
\hline $\mathrm{Ru}(2)$ & $8302(1)$ & $5950(1)$ & 4293(1) & $45(1)$ \\
\hline $\mathrm{Ru}(3)$ & $6342(1)$ & $9669(1)$ & $2115(1)$ & $47(1)$ \\
\hline $\mathrm{Cl}(1)$ & $7968(1)$ & $6206(1)$ & $543(1)$ & $76(1)$ \\
\hline $\mathrm{Cl}(2)$ & $9076(1)$ & $5199(1)$ & $4103(1)$ & $64(1)$ \\
\hline $\mathrm{Cl}(3)$ & $6969(1)$ & 9991(1) & $2887(1)$ & $68(1)$ \\
\hline $\mathrm{P}(1)$ & $6752(1)$ & $5081(1)$ & $526(1)$ & $65(1)$ \\
\hline $\mathrm{P}(2)$ & $8818(1)$ & $5314(1)$ & $1428(1)$ & $55(1)$ \\
\hline $\mathrm{P}(3)$ & $8954(1)$ & $6938(1)$ & $4308(1)$ & $52(1)$ \\
\hline $\mathrm{P}(4)$ & $7622(1)$ & 4966(1) & 4134(1) & $52(1)$ \\
\hline $\mathrm{P}(5)$ & $5513(1)$ & $9258(1)$ & 2399(1) & $52(1)$ \\
\hline$P(6)$ & 7231(1) & 9921(1) & $1856(1)$ & $62(1)$ \\
\hline C(1) & 7711(4) & $4350(5)$ & $1263(3)$ & $66(2)$ \\
\hline $\mathrm{C}(2)$ & 7733(4) & $6509(4)$ & $4436(3)$ & $51(2)$ \\
\hline$C(3)$ & $5923(4)$ & $9424(4)$ & $1544(3)$ & $60(2)$ \\
\hline $\mathrm{O}(1)$ & $7662(3)$ & $3867(4)$ & $1450(3)$ & $100(3)$ \\
\hline $\mathrm{O}(2)$ & $7379(3)$ & $6850(3)$ & $4541(2)$ & $80(2)$ \\
\hline $\mathrm{O}(3)$ & $5666(3)$ & $9266(4)$ & 1171(2) & $86(2)$ \\
\hline $\mathrm{N}(1)$ & $8180(3)$ & $4528(3)$ & 439(3) & $65(2)$ \\
\hline $\mathrm{N}(2)$ & $8739(3)$ & $5687(4)$ & $5037(2)$ & $68(2)$ \\
\hline $\mathrm{N}(3)$ & $6041(4)$ & 10793(4) & 2053(3) & $74(2)$ \\
\hline $\mathrm{C}(01)$ & $7350(3)$ & $6261(3)$ & 2111(3) & $41(2)$ \\
\hline $\mathrm{C}(02)$ & $7519(3)$ & $6179(4)$ & $2578(3)$ & $46(2)$ \\
\hline $\mathrm{C}(03)$ & 7398(3) & $6686(4)$ & 2872(3) & $46(2)$ \\
\hline $\mathrm{C}(04)$ & $7103(3)$ & $7299(4)$ & $2678(3)$ & $45(2)$ \\
\hline $\mathrm{C}(05)$ & $6946(3)$ & $7423(4)$ & $2212(3)$ & $45(2)$ \\
\hline $\mathrm{C}(06)$ & 7067(3) & $6893(4)$ & $1935(3)$ & $46(2)$ \\
\hline$C(11)$ & $7510(3)$ & $5763(4)$ & 1393(3) & $50(2)$ \\
\hline$C(12)$ & $7513(4)$ & $5715(4)$ & 1828(3) & $51(2)$ \\
\hline$C(21)$ & 7957(3) & $6143(4)$ & $3612(3)$ & $46(2)$ \\
\hline$C(22)$ & $7576(3)$ & $6607(4)$ & $3374(2)$ & $44(2)$ \\
\hline$C(31)$ & $6654(3)$ & $8668(4)$ & $2250(3)$ & $48(2)$ \\
\hline$C(32)$ & $6663(3)$ & $8087(4)$ & 2011(3) & $51(2)$ \\
\hline $\mathrm{C}(101)$ & $8162(5)$ & $4792(5)$ & $32(3)$ & $85(3)$ \\
\hline
\end{tabular}




\begin{tabular}{|c|c|c|c|c|}
\hline$C(102)$ & $8415(6)$ & $4462(6)$ & $-275(4)$ & 101(4) \\
\hline C(103) & $8709(6)$ & $3854(7)$ & $-161(5)$ & $104(4)$ \\
\hline C(104) & $8736(5)$ & $3575(6)$ & 253(4) & $98(4)$ \\
\hline$C(105)$ & $8463(5)$ & $3919(5)$ & $551(3)$ & 79(3) \\
\hline$C(201)$ & $9085(4)$ & $5114(6)$ & $5166(4)$ & $83(3)$ \\
\hline$C(202)$ & $9337(5)$ & $4952(8)$ & $5614(5)$ & $114(5)$ \\
\hline$C(203)$ & $9269(6)$ & $5394(11)$ & $5945(5)$ & $151(7)$ \\
\hline$C(204)$ & $8915(6)$ & $5973(9)$ & $5829(4)$ & $134(6)$ \\
\hline$C(205)$ & $8653(5)$ & $6117(6)$ & $5362(3)$ & $89(3)$ \\
\hline$C(301)$ & $6029(6)$ & $11173(5)$ & $2431(5)$ & $106(4)$ \\
\hline$C(302)$ & $5909(8)$ & 11873(7) & $2400(6)$ & $149(6)$ \\
\hline$C(303)$ & $5823(11)$ & $12219(7)$ & $1995(7)$ & 201(10) \\
\hline C(304) & $5793(7)$ & $11835(7)$ & $1616(5)$ & $141(6)$ \\
\hline$C(305)$ & $5906(5)$ & $11136(5)$ & $1668(4)$ & $95(4)$ \\
\hline $\mathrm{C}(111)$ & $6245(4)$ & $4523(5)$ & $751(4)$ & $77(3)$ \\
\hline$C(112)$ & $5732(6)$ & $4203(7)$ & $465(5)$ & $113(4)$ \\
\hline$C(113)$ & $5332(8)$ & $3819(10)$ & $624(7)$ & $147(6)$ \\
\hline$C(114)$ & $5406(7)$ & $3763(7)$ & $1073(8)$ & $139(6)$ \\
\hline$C(115)$ & $5907(6)$ & $4075(7)$ & $1385(5)$ & $117(4)$ \\
\hline$C(116)$ & $6314(5)$ & $4458(6)$ & $1212(4)$ & $88(3)$ \\
\hline $\mathrm{C}(121)$ & $6597(4)$ & $4733(4)$ & $-54(2)$ & $90(3)$ \\
\hline $\mathrm{C}(122)$ & $6843(4)$ & $4082(4)$ & $-110(3)$ & $98(4)$ \\
\hline$C(123)$ & $6734(5)$ & $3789(5)$ & $-543(4)$ & $148(6)$ \\
\hline $\mathrm{C}(124)$ & $6378(7)$ & $4147(7)$ & $-919(2)$ & $259(14)$ \\
\hline$C(125)$ & $6131(7)$ & $4797(6)$ & $-862(2)$ & $340(20)$ \\
\hline$C(126)$ & $6241(6)$ & $5090(4)$ & $-430(3)$ & $209(10)$ \\
\hline $\mathrm{C}(131)$ & $6369(5)$ & $5924(5)$ & $469(4)$ & $88(3)$ \\
\hline C(132) & $5957(7)$ & $6075(7)$ & $704(5)$ & $154(7)$ \\
\hline$C(133)$ & $5693(10)$ & $6731(9)$ & 691(7) & $254(15)$ \\
\hline C(134) & $5879(9)$ & $7258(10)$ & $452(7)$ & $204(14)$ \\
\hline$C(135)$ & $6271(8)$ & $7113(7)$ & 191(7) & $169(8)$ \\
\hline$C(136)$ & $6524(5)$ & $6454(6)$ & $213(5)$ & $121(5)$ \\
\hline$C(211)$ & $8989(4)$ & $4868(5)$ & 1979(3) & $60(2)$ \\
\hline$C(212)$ & $9004(4)$ & $4141(5)$ & 2003(3) & $72(3)$ \\
\hline$C(213)$ & $9151(5)$ & $3802(6)$ & $2423(4)$ & $86(3)$ \\
\hline$C(214)$ & $9246(5)$ & $4174(8)$ & $2810(4)$ & $99(4)$ \\
\hline$C(215)$ & $9206(5)$ & $4874(8)$ & $2797(4)$ & $98(4)$ \\
\hline$C(216)$ & $9081(4)$ & $5217(5)$ & $2382(3)$ & $76(3)$ \\
\hline
\end{tabular}




\begin{tabular}{|c|c|c|c|c|}
\hline$C(221)$ & $9088(4)$ & $6212(5)$ & $1598(3)$ & $64(2)$ \\
\hline$C(222)$ & $8708(5)$ & $6765(5)$ & $1600(4)$ & $87(3)$ \\
\hline$C(223)$ & $8928(6)$ & $7427(6)$ & $1741(4)$ & $106(4)$ \\
\hline$C(224)$ & $9537(7)$ & $7527(7)$ & $1887(5)$ & $118(4)$ \\
\hline$C(225)$ & $9934(6)$ & $6977(9)$ & 1901(5) & $135(5)$ \\
\hline$C(226)$ & $9713(6)$ & $6312(7)$ & $1761(4)$ & $109(4)$ \\
\hline$C(231)$ & $9424(4)$ & $5028(5)$ & 1171(3) & $63(2)$ \\
\hline$C(232)$ & $9453(5)$ & $5369(5)$ & $775(4)$ & $78(3)$ \\
\hline$C(233)$ & $9892(6)$ & $5187(6)$ & $556(4)$ & $97(4)$ \\
\hline$C(234)$ & $10304(6)$ & $4663(8)$ & $731(5)$ & $111(4)$ \\
\hline$C(235)$ & 10281(6) & 4307(8) & $1122(5)$ & $126(5)$ \\
\hline$C(236)$ & $9840(5)$ & $4511(6)$ & $1341(4)$ & $100(4)$ \\
\hline $\mathrm{C}(311)$ & $8531(4)$ & $7768(4)$ & $4162(3)$ & $55(2)$ \\
\hline$C(312)$ & $8228(4)$ & $8056(5)$ & $4458(3)$ & $71(3)$ \\
\hline$C(313)$ & $7873(5)$ & $8648(5)$ & $4339(4)$ & $79(3)$ \\
\hline$C(314)$ & $7813(5)$ & $8952(5)$ & $3923(4)$ & $79(3)$ \\
\hline$C(315)$ & $8091(4)$ & $8683(5)$ & $3633(4)$ & $77(3)$ \\
\hline$C(316)$ & $8454(4)$ & $8091(5)$ & $3747(3)$ & $64(2)$ \\
\hline$C(321)$ & $9453(4)$ & $6945(4)$ & $3915(3)$ & $60(2)$ \\
\hline$C(322)$ & $9258(6)$ & $6712(6)$ & $3483(4)$ & $108(4)$ \\
\hline$C(323)$ & $9602(7)$ & $6785(7)$ & $3181(5)$ & $129(5)$ \\
\hline $\mathrm{C}(324)$ & $10166(7)$ & $7065(8)$ & $3305(5)$ & $126(5)$ \\
\hline$C(325)$ & $10358(6)$ & $7310(10)$ & $3730(5)$ & $163(7)$ \\
\hline C(326) & $10005(6)$ & $7253(8)$ & $4045(5)$ & $146(6)$ \\
\hline$C(331)$ & $9521(4)$ & $7099(4)$ & $4856(3)$ & $55(2)$ \\
\hline C(332) & $9899(4)$ & $6536(5)$ & $5035(3)$ & $71(3)$ \\
\hline C(333) & $10322(5)$ & $6596(6)$ & $5456(4)$ & $95(4)$ \\
\hline C(334) & $10371(5)$ & $7212(6)$ & $5695(4)$ & $97(4)$ \\
\hline$C(335)$ & $10018(5)$ & $7758(6)$ & $5525(4)$ & $100(4)$ \\
\hline$C(336)$ & $9580(5)$ & $7714(5)$ & $5106(3)$ & $81(3)$ \\
\hline $\mathrm{C}(411)$ & $7790(4)$ & $4243(4)$ & $4540(3)$ & $58(2)$ \\
\hline$C(412)$ & $7856(6)$ & $3537(5)$ & $4433(4)$ & $104(4)$ \\
\hline$C(413)$ & $7962(8)$ & $3035(8)$ & $4753(7)$ & $153(6)$ \\
\hline C(414) & $8005(8)$ & $3195(9)$ & $5194(7)$ & $152(7)$ \\
\hline$C(415)$ & $7928(6)$ & $3885(9)$ & $5319(5)$ & $130(5)$ \\
\hline$C(416)$ & $7814(5)$ & $4410(6)$ & 4984(4) & $87(3)$ \\
\hline$C(421)$ & $6816(4)$ & $5117(4)$ & $4114(3)$ & $54(2)$ \\
\hline C(422) & $6460(4)$ & $4606(5)$ & $4224(4)$ & $84(3)$ \\
\hline
\end{tabular}




\begin{tabular}{|c|c|c|c|c|}
\hline$C(423)$ & $5849(5)$ & $4703(6)$ & $4189(4)$ & $95(4)$ \\
\hline C(424) & $5587(4)$ & $5327(6)$ & 4064(4) & $84(3)$ \\
\hline$C(425)$ & 5911(4) & $5842(5)$ & $3940(4)$ & $86(3)$ \\
\hline$C(426)$ & $6529(4)$ & $5739(5)$ & $3962(3)$ & $75(3)$ \\
\hline $\mathrm{C}(431)$ & $7549(4)$ & $4566(4)$ & $3572(3)$ & $61(2)$ \\
\hline C(432) & $7974(5)$ & $4135(5)$ & $3481(4)$ & $88(3)$ \\
\hline C(433) & $7903(6)$ & $3840(6)$ & $3052(5)$ & $104(4)$ \\
\hline C(434) & $7437(7)$ & $4008(7)$ & $2702(5)$ & $110(4)$ \\
\hline$C(435)$ & $7016(6)$ & $4492(6)$ & $2769(4)$ & $97(4)$ \\
\hline C(436) & $7064(5)$ & $4768(5)$ & $3210(3)$ & $73(3)$ \\
\hline$C(511)$ & $5727(4)$ & $8705(5)$ & 2904(3) & $64(2)$ \\
\hline $\mathrm{C}(512)$ & $6107(5)$ & $8911(8)$ & $3289(4)$ & $141(6)$ \\
\hline$C(513)$ & $6284(5)$ & $8502(10)$ & $3669(5)$ & $168(8)$ \\
\hline$C(514)$ & $6093(7)$ & $7850(8)$ & $3667(5)$ & $125(5)$ \\
\hline$C(515)$ & $5700(11)$ & $7622(7)$ & $3289(5)$ & $215(11)$ \\
\hline$C(516)$ & $5543(8)$ & $8042(6)$ & 2911(5) & $152(6)$ \\
\hline$C(521)$ & 4939(4) & $8712(4)$ & 2009(3) & $61(2)$ \\
\hline$C(522)$ & $5093(5)$ & $8198(6)$ & $1754(4)$ & $109(4)$ \\
\hline$C(523)$ & $4659(6)$ & $7759(7)$ & $1463(5)$ & $122(5)$ \\
\hline C(524) & $4068(7)$ & $7844(6)$ & $1436(4)$ & $107(4)$ \\
\hline$C(525)$ & $3881(5)$ & $8339(6)$ & $1705(5)$ & $106(4)$ \\
\hline$C(526)$ & $4317(5)$ & $8766(5)$ & 1991(4) & $90(3)$ \\
\hline$C(531)$ & $5036(4)$ & $9937(4)$ & $2555(3)$ & $54(2)$ \\
\hline$C(532)$ & $4969(5)$ & $10023(6)$ & 2977(4) & $98(3)$ \\
\hline$C(533)$ & $4571(7)$ & $10562(7)$ & $3060(5)$ & $126(5)$ \\
\hline$C(534)$ & $4269(6)$ & 10985(6) & $2725(6)$ & $104(4)$ \\
\hline$C(535)$ & $4333(5)$ & 10902(6) & $2310(5)$ & $103(4)$ \\
\hline$C(536)$ & $4713(5)$ & $10377(5)$ & $2224(4)$ & $91(3)$ \\
\hline$C(611)$ & $7135(4)$ & $9591(6)$ & $1278(3)$ & $71(3)$ \\
\hline$C(612)$ & $6780(6)$ & $9928(7)$ & $907(4)$ & $104(4)$ \\
\hline C(613) & $6662(7)$ & 9652(9) & $476(4)$ & $123(5)$ \\
\hline C(614) & $6890(7)$ & $9038(10)$ & $405(5)$ & $120(5)$ \\
\hline$C(615)$ & $7243(6)$ & $8676(8)$ & $758(5)$ & $116(5)$ \\
\hline$C(616)$ & $7372(5)$ & $8949(6)$ & 1201(4) & $91(3)$ \\
\hline$C(621)$ & $7984(4)$ & $9571(6)$ & 2171(4) & $87(3)$ \\
\hline$C(622)$ & $8075(5)$ & $8950(8)$ & 2393(4) & $134(6)$ \\
\hline$C(623)$ & $8639(6)$ & $8655(10)$ & $2578(4)$ & $176(8)$ \\
\hline$C(624)$ & $9142(7)$ & $9016(12)$ & $2539(7)$ & $229(15)$ \\
\hline
\end{tabular}




\begin{tabular}{lcccr}
$\mathrm{C}(625)$ & $9062(9)$ & $9665(12)$ & $2341(10)$ & $310(20)$ \\
$\mathrm{C}(626)$ & $8494(6)$ & $9908(9)$ & $2128(8)$ & $221(11)$ \\
$\mathrm{C}(631)$ & $7434(5)$ & $10851(5)$ & $1833(4)$ & $96(4)$ \\
$\mathrm{C}(632)$ & $7415(5)$ & $11276(5)$ & $2183(4)$ & $117(4)$ \\
$\mathrm{C}(633)$ & $7607(7)$ & $11958(6)$ & $2195(5)$ & $152(6)$ \\
$\mathrm{C}(634)$ & $7828(9)$ & $12220(9)$ & $1859(6)$ & $211(10)$ \\
$\mathrm{C}(635)$ & $7817(15)$ & $11812(10)$ & $1499(8)$ & $370(30)$ \\
$\mathrm{C}(636)$ & $7624(10)$ & $11138(9)$ & $1494(5)$ & $235(12)$ \\
$\mathrm{C}(41)$ & $12023(8)$ & $6224(10)$ & $1469(8)$ & $230(30)$ \\
$\mathrm{Cl}(4)$ & $12299(6)$ & $7011(5)$ & $1453(4)$ & $208(5)$ \\
$\mathrm{Cl}(5)$ & $11292(4)$ & $6153(6)$ & $1429(3)$ & $192(4)$ \\
$\mathrm{C}(42)$ & $4092(12)$ & $6766(14)$ & $4515(8)$ & $129(9)$ \\
$\mathrm{Cl}(6)$ & $3673(11)$ & $7162(13)$ & $4785(9)$ & $446(14)$ \\
$\mathrm{Cl}(7)$ & $4679(10)$ & $6376(11)$ & $4825(8)$ & $391(11)$ \\
\hline
\end{tabular}

$\mathrm{U}(\mathrm{eq})$ is defined as one third of the trace of the orthogonalized Uij tensor. 
Table S2. Bond lengths $[\AA]$ for $1,3,5-\left[\mathrm{Cl}(\mathrm{CO})(\mathrm{Py})\left(\mathrm{PPh}_{3}\right)_{2} \mathrm{RuCH}=\mathrm{CH}\right]_{3} \mathrm{C}_{6} \mathrm{H}_{3} \cdot \mathrm{CH}_{2} \mathrm{Cl}_{2}\left(\mathbf{5}^{\circ} \mathrm{CH}_{2} \mathrm{Cl}_{2}\right)$.

\begin{tabular}{|c|c|c|c|c|c|}
\hline $\mathrm{Ru}(1)-\mathrm{P}(1)$ & $2.397(2)$ & $\mathrm{Ru}(2)-\mathrm{P}(4)$ & $2.395(2)$ & $\mathrm{Ru}(3)-\mathrm{P}(5)$ & $2.404(2)$ \\
\hline $\mathrm{Ru}(1)-\mathrm{P}(2)$ & $2.389(2)$ & $\mathrm{Ru}(2)-\mathrm{P}(3)$ & $2.389(2)$ & $\mathrm{Ru}(3)-\mathrm{P}(6)$ & $2.404(2)$ \\
\hline $\mathrm{Ru}(1)-\mathrm{Cl}(1)$ & $2.475(2)$ & $\mathrm{Ru}(2)-\mathrm{Cl}(2)$ & $2.454(2)$ & $\mathrm{Ru}(3)-\mathrm{Cl}(3)$ & $2.474(2)$ \\
\hline $\mathrm{Ru}(1)-\mathrm{C}(1)$ & $1.809(10)$ & $\mathrm{Ru}(2)-\mathrm{C}(2)$ & $1.814(9)$ & $\mathrm{Ru}(3)-\mathrm{C}(3)$ & $1.806(10)$ \\
\hline $\mathrm{Ru}(1)-\mathrm{C}(11)$ & $2.050(8)$ & $\mathrm{Ru}(2)-\mathrm{C}(21)$ & $2.043(7)$ & $\operatorname{Ru}(3)-C(31)$ & $2.038(7)$ \\
\hline $\mathrm{Ru}(1)-\mathrm{N}(1)$ & $2.276(7)$ & $\mathrm{Ru}(2)-\mathrm{N}(2)$ & $2.271(7)$ & $\mathrm{Ru}(3)-\mathrm{N}(3)$ & $2.243(7)$ \\
\hline $\mathrm{C}(1)-\mathrm{O}(1)$ & $1.105(10)$ & $\mathrm{C}(2)-\mathrm{O}(2)$ & $1.145(9)$ & $\mathrm{C}(3)-\mathrm{O}(3)$ & $1.167(10)$ \\
\hline$C(11)-C(12)$ & $1.326(10)$ & $\mathrm{C}(21)-\mathrm{C}(22)$ & $1.311(9)$ & $\mathrm{C}(31)-\mathrm{C}(32)$ & $1.330(10)$ \\
\hline $\mathrm{C}(01)-\mathrm{C}(02)$ & $1.378(10)$ & $\mathrm{C}(02)-\mathrm{C}(03)$ & $1.393(10)$ & $\mathrm{C}(03)-\mathrm{C}(04)$ & $1.397(10)$ \\
\hline $\mathrm{C}(04)-\mathrm{C}(05)$ & $1.386(10)$ & $\mathrm{C}(05)-\mathrm{C}(06)$ & $1.392(10)$ & $\mathrm{C}(01)-\mathrm{C}(06)$ & $1.402(9)$ \\
\hline $\mathrm{C}(01)-\mathrm{C}(12)$ & $1.461(10)$ & $\mathrm{C}(03)-\mathrm{C}(22)$ & $1.479(10)$ & $\mathrm{C}(05)-\mathrm{C}(32)$ & $1.476(10)$ \\
\hline $\mathrm{N}(1)-\mathrm{C}(105)$ & $1.326(10)$ & $\mathrm{N}(2)-\mathrm{C}(205)$ & $1.340(11)$ & $\mathrm{N}(3)-\mathrm{C}(305)$ & $1.304(12)$ \\
\hline $\mathrm{N}(1)-\mathrm{C}(101)$ & $1.329(11)$ & $\mathrm{N}(2)-\mathrm{C}(201)$ & $1.341(11)$ & $\mathrm{N}(3)-\mathrm{C}(301)$ & $1.366(13)$ \\
\hline$C(101)-C(102)$ & $1.373(13)$ & $\mathrm{C}(201)-\mathrm{C}(202)$ & $1.367(14)$ & $\mathrm{C}(301)-\mathrm{C}(302)$ & $1.361(15)$ \\
\hline$C(102)-C(103)$ & $1.337(15)$ & $C(202)-C(203)$ & $1.354(18)$ & $C(302)-C(303)$ & $1.365(19)$ \\
\hline$C(103)-C(104)$ & $1.353(15)$ & $C(203)-C(204)$ & $1.354(19)$ & $\mathrm{C}(303)-\mathrm{C}(304)$ & $1.353(19)$ \\
\hline$C(104)-C(105)$ & $1.391(13)$ & $C(204)-C(205)$ & $1.414(15)$ & $C(304)-C(305)$ & $1.357(14)$ \\
\hline $\mathrm{P}(1)-\mathrm{C}(131)$ & $1.814(10)$ & $\mathrm{P}(3)-\mathrm{C}(331)$ & $1.840(8)$ & $\mathrm{P}(5)-\mathrm{C}(511)$ & $1.818(9)$ \\
\hline $\mathrm{P}(1)-\mathrm{C}(121)$ & $1.829(6)$ & $\mathrm{P}(3)-\mathrm{C}(311)$ & $1.842(9)$ & $P(5)-C(531)$ & $1.830(8)$ \\
\hline $\mathrm{P}(1)-\mathrm{C}(111)$ & $1.829(11)$ & $\mathrm{P}(3)-\mathrm{C}(321)$ & $1.854(9)$ & $P(5)-C(521)$ & $1.834(9)$ \\
\hline $\mathrm{P}(2)-\mathrm{C}(211)$ & $1.826(9)$ & $\mathrm{P}(4)-\mathrm{C}(411)$ & $1.822(9)$ & $\mathrm{P}(6)-\mathrm{C}(611)$ & $1.826(10)$ \\
\hline $\mathrm{P}(2)-\mathrm{C}(231)$ & $1.839(9)$ & $\mathrm{P}(4)-\mathrm{C}(421)$ & $1.836(8)$ & $P(6)-C(631)$ & $1.838(10)$ \\
\hline $\mathrm{P}(2)-\mathrm{C}(221)$ & $1.846(9)$ & $\mathrm{P}(4)-\mathrm{C}(431)$ & $1.840(9)$ & $\mathrm{P}(6)-\mathrm{C}(621)$ & $1.844(11)$ \\
\hline$C(111)-C(116)$ & $1.374(14)$ & $\mathrm{C}(311)-\mathrm{C}(316)$ & $1.374(11)$ & $\mathrm{C}(511)-\mathrm{C}(512)$ & $1.315(11)$ \\
\hline $\mathrm{C}(111)-\mathrm{C}(112)$ & $1.393(14)$ & $\mathrm{C}(311)-\mathrm{C}(312)$ & $1.385(11)$ & $\mathrm{C}(511)-\mathrm{C}(516)$ & $1.334(11)$ \\
\hline $\mathrm{C}(112)-\mathrm{C}(113)$ & $1.352(18)$ & $\mathrm{C}(312)-\mathrm{C}(313)$ & $1.377(12)$ & $\mathrm{C}(512)-\mathrm{C}(513)$ & $1.365(11)$ \\
\hline $\mathrm{C}(113)-\mathrm{C}(114)$ & $1.34(2)$ & $\mathrm{C}(313)-\mathrm{C}(314)$ & $1.365(13)$ & $\mathrm{C}(513)-\mathrm{C}(514)$ & $1.316(12)$ \\
\hline$C(114)-C(115)$ & $1.402(19)$ & $\mathrm{C}(314)-\mathrm{C}(315)$ & $1.320(13)$ & $\mathrm{C}(514)-\mathrm{C}(515)$ & $1.327(12)$ \\
\hline$C(115)-C(116)$ & $1.388(15)$ & $C(315)-C(316)$ & $1.386(12)$ & $\mathrm{C}(515)-\mathrm{C}(516)$ & $1.367(12)$ \\
\hline $\mathrm{C}(121)-\mathrm{C}(122)$ & 1.3900 & $\mathrm{C}(321)-\mathrm{C}(326)$ & $1.343(13)$ & $\mathrm{C}(521)-\mathrm{C}(522)$ & $1.353(12)$ \\
\hline$C(121)-C(126)$ & 1.3900 & $\mathrm{C}(321)-\mathrm{C}(322)$ & $1.346(12)$ & $\mathrm{C}(521)-\mathrm{C}(526)$ & $1.402(12)$ \\
\hline $\mathrm{C}(122)-\mathrm{C}(123)$ & 1.3900 & $\mathrm{C}(322)-\mathrm{C}(323)$ & $1.363(14)$ & $C(522)-C(523)$ & $1.409(15)$ \\
\hline $\mathrm{C}(123)-\mathrm{C}(124)$ & 1.3900 & $\mathrm{C}(323)-\mathrm{C}(324)$ & $1.344(16)$ & $\mathrm{C}(523)-\mathrm{C}(524)$ & $1.332(15)$ \\
\hline $\mathrm{C}(124)-\mathrm{C}(125)$ & 1.3900 & $\mathrm{C}(324)-\mathrm{C}(325)$ & $1.335(17)$ & $\mathrm{C}(524)-\mathrm{C}(525)$ & $1.387(15)$ \\
\hline$C(125)-C(126)$ & 1.3900 & $\mathrm{C}(325)-\mathrm{C}(326)$ & $1.410(16)$ & $\mathrm{C}(525)-\mathrm{C}(526)$ & $1.392(14)$ \\
\hline
\end{tabular}




\begin{tabular}{|c|c|c|c|c|c|}
\hline $\mathrm{C}(131)-\mathrm{C}(132)$ & $1.353(12)$ & $\mathrm{C}(331)-\mathrm{C}(336)$ & $1.383(11)$ & $\mathrm{C}(531)-\mathrm{C}(532)$ & $1.343(12)$ \\
\hline $\mathrm{C}(131)-\mathrm{C}(136)$ & $1.379(11)$ & $\mathrm{C}(331)-\mathrm{C}(332)$ & $1.389(11)$ & $C(531)-C(536)$ & $1.364(12)$ \\
\hline$C(132)-C(133)$ & $1.383(12)$ & $C(332)-C(333)$ & $1.385(12)$ & $C(532)-C(533)$ & $1.435(15)$ \\
\hline $\mathrm{C}(133)-\mathrm{C}(134)$ & $1.372(14)$ & $\mathrm{C}(333)-\mathrm{C}(334)$ & $1.368(14)$ & $C(533)-C(534)$ & $1.336(16)$ \\
\hline $\mathrm{C}(134)-\mathrm{C}(135)$ & $1.369(14)$ & $\mathrm{C}(334)-\mathrm{C}(335)$ & $1.331(13)$ & $C(534)-C(535)$ & $1.320(16)$ \\
\hline$C(135)-C(136)$ & $1.377(12)$ & $\mathrm{C}(335)-\mathrm{C}(336)$ & $1.393(13)$ & $C(535)-C(536)$ & $1.389(13)$ \\
\hline$C(211)-C(216)$ & $1.362(12)$ & $C(411)-C(416)$ & $1.375(13)$ & $\mathrm{C}(611)-\mathrm{C}(612)$ & $1.359(14)$ \\
\hline $\mathrm{C}(211)-\mathrm{C}(212)$ & $1.387(11)$ & $\mathrm{C}(411)-\mathrm{C}(412)$ & $1.401(12)$ & $C(611)-C(616)$ & $1.383(13)$ \\
\hline$C(212)-C(213)$ & $1.390(13)$ & $\mathrm{C}(412)-\mathrm{C}(413)$ & $1.341(16)$ & $\mathrm{C}(612)-\mathrm{C}(613)$ & $1.371(16)$ \\
\hline $\mathrm{C}(213)-\mathrm{C}(214)$ & $1.342(15)$ & $\mathrm{C}(413)-\mathrm{C}(414)$ & $1.36(2)$ & $C(613)-C(614)$ & $1.321(18)$ \\
\hline$C(214)-C(215)$ & $1.339(15)$ & $\mathrm{C}(414)-\mathrm{C}(415)$ & $1.393(19)$ & $C(614)-C(615)$ & $1.346(18)$ \\
\hline$C(215)-C(216)$ & $1.384(14)$ & $\mathrm{C}(415)-\mathrm{C}(416)$ & $1.403(15)$ & $C(615)-C(616)$ & $1.399(15)$ \\
\hline $\mathrm{C}(221)-\mathrm{C}(222)$ & $1.362(12)$ & $\mathrm{C}(421)-\mathrm{C}(422)$ & $1.363(12)$ & $\mathrm{C}(621)-\mathrm{C}(622)$ & $1.350(11)$ \\
\hline$C(221)-C(226)$ & $1.384(13)$ & $\mathrm{C}(421)-\mathrm{C}(426)$ & $1.373(11)$ & $C(621)-C(626)$ & $1.362(12)$ \\
\hline$C(222)-C(223)$ & $1.383(13)$ & $\mathrm{C}(422)-\mathrm{C}(423)$ & $1.374(13)$ & $\mathrm{C}(622)-\mathrm{C}(623)$ & $1.373(12)$ \\
\hline $\mathrm{C}(223)-\mathrm{C}(224)$ & $1.348(16)$ & $\mathrm{C}(423)-\mathrm{C}(424)$ & $1.338(13)$ & $\mathrm{C}(623)-\mathrm{C}(624)$ & $1.364(13)$ \\
\hline $\mathrm{C}(224)-\mathrm{C}(225)$ & $1.375(17)$ & $\mathrm{C}(424)-\mathrm{C}(425)$ & $1.343(13)$ & $\mathrm{C}(624)-\mathrm{C}(625)$ & $1.367(14)$ \\
\hline$C(225)-C(226)$ & $1.387(16)$ & $\mathrm{C}(425)-\mathrm{C}(426)$ & $1.398(12)$ & $\mathrm{C}(625)-\mathrm{C}(626)$ & $1.359(14)$ \\
\hline$C(231)-C(236)$ & $1.366(13)$ & $\mathrm{C}(431)-\mathrm{C}(432)$ & $1.351(12)$ & $\mathrm{C}(631)-\mathrm{C}(636)$ & $1.338(11)$ \\
\hline $\mathrm{C}(231)-\mathrm{C}(232)$ & $1.386(12)$ & $\mathrm{C}(431)-\mathrm{C}(436)$ & $1.388(12)$ & $\mathrm{C}(631)-\mathrm{C}(632)$ & $1.347(11)$ \\
\hline $\mathrm{C}(232)-\mathrm{C}(233)$ & $1.385(13)$ & $\mathrm{C}(432)-\mathrm{C}(433)$ & $1.390(15)$ & $\mathrm{C}(632)-\mathrm{C}(633)$ & $1.368(11)$ \\
\hline $\mathrm{C}(233)-\mathrm{C}(234)$ & $1.374(16)$ & $\mathrm{C}(433)-\mathrm{C}(434)$ & $1.323(17)$ & $\mathrm{C}(633)-\mathrm{C}(634)$ & $1.350(12)$ \\
\hline$C(234)-C(235)$ & $1.383(16)$ & $\mathrm{C}(434)-\mathrm{C}(435)$ & $1.383(16)$ & $\mathrm{C}(634)-\mathrm{C}(635)$ & $1.339(13)$ \\
\hline$C(235)-C(236)$ & $1.398(15)$ & $\mathrm{C}(435)-\mathrm{C}(436)$ & $1.419(13)$ & $\mathrm{C}(635)-\mathrm{C}(636)$ & $1.356(12)$ \\
\hline $\mathrm{C}(41)-\mathrm{Cl}(4)$ & $1.631(16)$ & $\mathrm{C}(41)-\mathrm{Cl}(5)$ & $1.636(17)$ & $\mathrm{C}(42)-\mathrm{Cl}(6)$ & $1.603(17)$ \\
\hline $\mathrm{C}(42)-\mathrm{Cl}(7)$ & $1.596(17)$ & & & & \\
\hline
\end{tabular}


Table S3. Bond angles [ $\left.{ }^{\circ}\right]$ for $1,3,5-\left[\mathrm{Cl}(\mathrm{CO})(\mathrm{Py})\left(\mathrm{PPh}_{3}\right)_{2} \mathrm{RuCH}=\mathrm{CH}\right]_{3} \mathrm{C}_{6} \mathrm{H}_{3} \cdot \mathrm{CH}_{2} \mathrm{Cl}_{2}\left(\mathbf{5}^{\circ} \mathrm{CH}_{2} \mathrm{Cl}_{2}\right)$.

\begin{tabular}{|c|c|c|c|}
\hline $\mathrm{P}(2)-\mathrm{Ru}(1)-\mathrm{P}(1)$ & $172.18(8)$ & $\mathrm{C}(1)-\mathrm{Ru}(1)-\mathrm{Cl}(1)$ & $178.2(3)$ \\
\hline $\mathrm{C}(11)-\mathrm{Ru}(1)-\mathrm{N}(1)$ & $174.2(3)$ & $\mathrm{C}(1)-\mathrm{Ru}(1)-\mathrm{C}(11)$ & $91.9(4)$ \\
\hline $\mathrm{C}(1)-\mathrm{Ru}(1)-\mathrm{N}(1)$ & $93.5(3)$ & $\mathrm{C}(1)-\mathrm{Ru}(1)-\mathrm{P}(1)$ & $90.4(3)$ \\
\hline $\mathrm{C}(11)-\mathrm{Ru}(1)-\mathrm{P}(1)$ & $85.5(2)$ & $\mathrm{C}(1)-\mathrm{Ru}(1)-\mathrm{P}(2)$ & $91.8(3)$ \\
\hline $\mathrm{C}(11)-\mathrm{Ru}(1)-\mathrm{P}(2)$ & $86.9(2)$ & $\mathrm{N}(1)-\mathrm{Ru}(1)-\mathrm{P}(1)$ & $96.43(19)$ \\
\hline N(1)-Ru(1)-P(2) & $90.90(19)$ & $\mathrm{P}(1)-\mathrm{Ru}(1)-\mathrm{Cl}(1)$ & $91.35(9)$ \\
\hline $\mathrm{P}(2)-\mathrm{Ru}(1)-\mathrm{Cl}(1)$ & $86.34(8)$ & $\mathrm{C}(11)-\mathrm{Ru}(1)-\mathrm{Cl}(1)$ & $87.8(2)$ \\
\hline $\mathrm{N}(1)-\mathrm{Ru}(1)-\mathrm{Cl}(1)$ & $86.7(2)$ & $\mathrm{O}(1)-\mathrm{C}(1)-\mathrm{Ru}(1)$ & $178.6(10)$ \\
\hline $\mathrm{P}(3)-\mathrm{Ru}(2)-\mathrm{P}(4)$ & $169.78(8)$ & $\mathrm{C}(2)-\mathrm{Ru}(2)-\mathrm{Cl}(2)$ & $179.7(3)$ \\
\hline $\mathrm{C}(21)-\mathrm{Ru}(2)-\mathrm{N}(2)$ & $175.8(3)$ & $\mathrm{C}(2)-\mathrm{Ru}(2)-\mathrm{C}(21)$ & $91.9(3)$ \\
\hline $\mathrm{C}(2)-\mathrm{Ru}(2)-\mathrm{N}(2)$ & $92.3(3)$ & $\mathrm{C}(2)-\mathrm{Ru}(2)-\mathrm{P}(3)$ & $90.5(2)$ \\
\hline $\mathrm{C}(21)-\mathrm{Ru}(2)-\mathrm{P}(3)$ & $86.8(2)$ & $\mathrm{C}(2)-\mathrm{Ru}(2)-\mathrm{P}(4)$ & $92.7(2)$ \\
\hline $\mathrm{N}(2)-\mathrm{Ru}(2)-\mathrm{P}(4)$ & $96.5(2)$ & $\mathrm{C}(21)-\mathrm{Ru}(2)-\mathrm{P}(4)$ & $83.4(2)$ \\
\hline $\mathrm{N}(2)-\mathrm{Ru}(2)-\mathrm{P}(3)$ & $93.05(19)$ & $\mathrm{P}(3)-\mathrm{Ru}(2)-\mathrm{Cl}(2)$ & $89.16(8)$ \\
\hline $\mathrm{P}(4)-\mathrm{Ru}(2)-\mathrm{Cl}(2)$ & $87.63(7)$ & $\mathrm{C}(21)-\mathrm{Ru}(2)-\mathrm{Cl}(2)$ & $88.2(2)$ \\
\hline $\mathrm{N}(2)-\mathrm{Ru}(2)-\mathrm{Cl}(2)$ & $87.6(2)$ & $\mathrm{O}(2)-\mathrm{C}(2)-\mathrm{Ru}(2)$ & $177.4(8)$ \\
\hline$P(6)-R u(3)-P(5)$ & $172.09(8)$ & $\mathrm{C}(3)-\mathrm{Ru}(3)-\mathrm{Cl}(3)$ & $177.0(3)$ \\
\hline $\mathrm{C}(31)-\mathrm{Ru}(3)-\mathrm{N}(3)$ & $173.4(3)$ & $\mathrm{C}(3)-\mathrm{Ru}(3)-\mathrm{C}(31)$ & $91.0(3)$ \\
\hline $\mathrm{C}(3)-\mathrm{Ru}(3)-\mathrm{N}(3)$ & $95.4(3)$ & $\mathrm{C}(3)-\mathrm{Ru}(3)-\mathrm{P}(5)$ & $90.1(3)$ \\
\hline $\mathrm{C}(31)-\mathrm{Ru}(3)-\mathrm{P}(5)$ & $83.4(2)$ & $\mathrm{C}(3)-\mathrm{Ru}(3)-\mathrm{P}(6)$ & $90.4(3)$ \\
\hline N(3)-Ru(3)-P(6) & $92.6(2)$ & $\mathrm{C}(31)-\mathrm{Ru}(3)-\mathrm{P}(6)$ & $88.7(2)$ \\
\hline $\mathrm{N}(3)-\mathrm{Ru}(3)-\mathrm{P}(5)$ & $95.2(2)$ & $\mathrm{P}(5)-\mathrm{Ru}(3)-\mathrm{Cl}(3)$ & $92.59(8)$ \\
\hline $\mathrm{P}(6)-\mathrm{Ru}(3)-\mathrm{Cl}(3)$ & $86.75(8)$ & $\mathrm{C}(31)-\mathrm{Ru}(3)-\mathrm{Cl}(3)$ & $87.8(2)$ \\
\hline $\mathrm{N}(3)-\mathrm{Ru}(3)-\mathrm{Cl}(3)$ & $85.8(2)$ & $\mathrm{O}(3)-\mathrm{C}(3)-\mathrm{Ru}(3)$ & $178.2(7)$ \\
\hline $\mathrm{C}(02)-\mathrm{C}(01)-\mathrm{C}(12)$ & $118.9(6)$ & $\mathrm{C}(06)-\mathrm{C}(01)-\mathrm{C}(12)$ & $123.2(7)$ \\
\hline $\mathrm{C}(02)-\mathrm{C}(03)-\mathrm{C}(22)$ & $123.1(7)$ & $\mathrm{C}(04)-\mathrm{C}(03)-\mathrm{C}(22)$ & $119.3(7)$ \\
\hline $\mathrm{C}(04)-\mathrm{C}(05)-\mathrm{C}(32)$ & $122.3(7)$ & $\mathrm{C}(06)-\mathrm{C}(05)-\mathrm{C}(32)$ & $120.4(7)$ \\
\hline$C(11)-C(12)-C(01)$ & $127.3(7)$ & $\mathrm{C}(12)-\mathrm{C}(11)-\mathrm{Ru}(1)$ & $134.5(6)$ \\
\hline $\mathrm{C}(21)-\mathrm{C}(22)-\mathrm{C}(03)$ & $126.7(7)$ & $\mathrm{C}(22)-\mathrm{C}(21)-\mathrm{Ru}(2)$ & $134.4(6)$ \\
\hline $\mathrm{C}(31)-\mathrm{C}(32)-\mathrm{C}(05)$ & $123.8(7)$ & $\mathrm{C}(32)-\mathrm{C}(31)-\mathrm{Ru}(3)$ & $136.5(6)$ \\
\hline $\mathrm{C}(111)-\mathrm{P}(1)-\mathrm{Ru}(1)$ & $117.2(3)$ & $\mathrm{C}(211)-\mathrm{P}(2)-\mathrm{Ru}(1)$ & $115.0(3)$ \\
\hline $\mathrm{C}(121)-\mathrm{P}(1)-\mathrm{Ru}(1)$ & 116.1(3) & $\mathrm{C}(231)-\mathrm{P}(2)-\mathrm{Ru}(1)$ & $113.9(3)$ \\
\hline $\mathrm{C}(131)-\mathrm{P}(1)-\mathrm{Ru}(1)$ & $113.5(3)$ & $\mathrm{C}(221)-\mathrm{P}(2)-\mathrm{Ru}(1)$ & $119.9(3)$ \\
\hline $\mathrm{C}(331)-\mathrm{P}(3)-\mathrm{Ru}(2)$ & $115.3(3)$ & $\mathrm{C}(411)-\mathrm{P}(4)-\mathrm{Ru}(2)$ & $116.7(3)$ \\
\hline $\mathrm{C}(311)-\mathrm{P}(3)-\mathrm{Ru}(2)$ & 113.1(3) & $\mathrm{C}(421)-\mathrm{P}(4)-\mathrm{Ru}(2)$ & $117.8(3)$ \\
\hline
\end{tabular}




\begin{tabular}{|c|c|c|c|}
\hline $\mathrm{C}(321)-\mathrm{P}(3)-\mathrm{Ru}(2)$ & $118.4(3)$ & $\mathrm{C}(431)-\mathrm{P}(4)-\mathrm{Ru}(2)$ & $113.8(3)$ \\
\hline $\mathrm{C}(511)-\mathrm{P}(5)-\mathrm{Ru}(3)$ & $115.8(3)$ & $\mathrm{C}(611)-\mathrm{P}(6)-\mathrm{Ru}(3)$ & $110.7(3)$ \\
\hline $\mathrm{C}(531)-\mathrm{P}(5)-\mathrm{Ru}(3)$ & $115.9(3)$ & $\mathrm{C}(631)-\mathrm{P}(6)-\mathrm{Ru}(3)$ & $116.5(4)$ \\
\hline $\mathrm{C}(521)-\mathrm{P}(5)-\mathrm{Ru}(3)$ & $116.1(3)$ & $\mathrm{C}(621)-\mathrm{P}(6)-\mathrm{Ru}(3)$ & $119.9(4)$ \\
\hline $\mathrm{C}(105)-\mathrm{N}(1)-\mathrm{Ru}(1)$ & $120.7(6)$ & $\mathrm{C}(101)-\mathrm{N}(1)-\mathrm{Ru}(1)$ & $121.5(6)$ \\
\hline $\mathrm{C}(205)-\mathrm{N}(2)-\mathrm{Ru}(2)$ & $119.3(7)$ & $\mathrm{C}(201)-\mathrm{N}(2)-\mathrm{Ru}(2)$ & $122.4(7)$ \\
\hline $\mathrm{C}(305)-\mathrm{N}(3)-\mathrm{Ru}(3)$ & $123.2(7)$ & $\mathrm{C}(301)-\mathrm{N}(3)-\mathrm{Ru}(3)$ & $120.6(7)$ \\
\hline $\mathrm{C}(02)-\mathrm{C}(01)-\mathrm{C}(06)$ & $117.7(7)$ & $\mathrm{C}(05)-\mathrm{C}(04)-\mathrm{C}(03)$ & $122.6(7)$ \\
\hline $\mathrm{C}(01)-\mathrm{C}(02)-\mathrm{C}(03)$ & $122.4(7)$ & $\mathrm{C}(04)-\mathrm{C}(05)-\mathrm{C}(06)$ & $117.3(6)$ \\
\hline $\mathrm{C}(02)-\mathrm{C}(03)-\mathrm{C}(04)$ & $117.6(7)$ & $\mathrm{C}(05)-\mathrm{C}(06)-\mathrm{C}(01)$ & $122.4(7)$ \\
\hline $\mathrm{C}(131)-\mathrm{P}(1)-\mathrm{C}(121)$ & $105.4(4)$ & $\mathrm{C}(211)-\mathrm{P}(2)-\mathrm{C}(231)$ & $104.6(4)$ \\
\hline $\mathrm{C}(131)-\mathrm{P}(1)-\mathrm{C}(111)$ & $102.6(5)$ & $\mathrm{C}(211)-\mathrm{P}(2)-\mathrm{C}(221)$ & $101.6(4)$ \\
\hline $\mathrm{C}(121)-\mathrm{P}(1)-\mathrm{C}(111)$ & $100.0(5)$ & $\mathrm{C}(231)-\mathrm{P}(2)-\mathrm{C}(221)$ & $99.5(4)$ \\
\hline $\mathrm{C}(331)-\mathrm{P}(3)-\mathrm{C}(311)$ & $105.5(4)$ & $\mathrm{C}(411)-\mathrm{P}(4)-\mathrm{C}(421)$ & $99.6(4)$ \\
\hline $\mathrm{C}(331)-\mathrm{P}(3)-\mathrm{C}(321)$ & $100.7(4)$ & $\mathrm{C}(411)-\mathrm{P}(4)-\mathrm{C}(431)$ & $105.5(4)$ \\
\hline $\mathrm{C}(311)-\mathrm{P}(3)-\mathrm{C}(321)$ & $102.0(4)$ & $\mathrm{C}(421)-\mathrm{P}(4)-\mathrm{C}(431)$ & $101.2(4)$ \\
\hline $\mathrm{C}(511)-\mathrm{P}(5)-\mathrm{C}(531)$ & $103.5(4)$ & $\mathrm{C}(611)-\mathrm{P}(6)-\mathrm{C}(631)$ & $105.2(5)$ \\
\hline $\mathrm{C}(511)-\mathrm{P}(5)-\mathrm{C}(521)$ & $101.6(4)$ & $\mathrm{C}(611)-\mathrm{P}(6)-\mathrm{C}(621)$ & $103.4(5)$ \\
\hline $\mathrm{C}(531)-\mathrm{P}(5)-\mathrm{C}(521)$ & $101.7(4)$ & $\mathrm{C}(631)-\mathrm{P}(6)-\mathrm{C}(621)$ & $99.4(5)$ \\
\hline $\mathrm{C}(105)-\mathrm{N}(1)-\mathrm{C}(101)$ & $117.6(8)$ & $\mathrm{N}(1)-\mathrm{C}(101)-\mathrm{C}(102)$ & $123.2(10)$ \\
\hline $\mathrm{C}(103)-\mathrm{C}(102)-\mathrm{C}(101)$ & $119.4(11)$ & $C(103)-C(104)-C(105)$ & $120.3(10)$ \\
\hline $\mathrm{C}(102)-\mathrm{C}(103)-\mathrm{C}(104)$ & $118.5(11)$ & $\mathrm{N}(1)-\mathrm{C}(105)-\mathrm{C}(104)$ & $121.0(10)$ \\
\hline $\mathrm{C}(205)-\mathrm{N}(2)-\mathrm{C}(201)$ & 118.3(9) & $\mathrm{N}(2)-\mathrm{C}(201)-\mathrm{C}(202)$ & $122.1(11)$ \\
\hline $\mathrm{C}(203)-\mathrm{C}(202)-\mathrm{C}(201)$ & $120.1(13)$ & $C(203)-C(204)-C(205)$ & $118.7(12)$ \\
\hline $\mathrm{C}(204)-\mathrm{C}(203)-\mathrm{C}(202)$ & $119.5(13)$ & $\mathrm{N}(2)-\mathrm{C}(205)-\mathrm{C}(204)$ & $121.2(11)$ \\
\hline $\mathrm{C}(305)-\mathrm{N}(3)-\mathrm{C}(301)$ & $116.0(9)$ & $\mathrm{C}(302)-\mathrm{C}(301)-\mathrm{N}(3)$ & $120.6(12)$ \\
\hline $\mathrm{C}(301)-\mathrm{C}(302)-\mathrm{C}(303)$ & $121.0(14)$ & $\mathrm{C}(303)-\mathrm{C}(304)-\mathrm{C}(305)$ & $117.8(14)$ \\
\hline $\mathrm{C}(304)-\mathrm{C}(303)-\mathrm{C}(302)$ & 118.1(13) & $\mathrm{N}(3)-\mathrm{C}(305)-\mathrm{C}(304)$ & $126.1(12)$ \\
\hline $\mathrm{C}(116)-\mathrm{C}(111)-\mathrm{P}(1)$ & $121.6(8)$ & $\mathrm{C}(216)-\mathrm{C}(211)-\mathrm{C}(212)$ & $116.4(9)$ \\
\hline $\mathrm{C}(112)-\mathrm{C}(111)-\mathrm{P}(1)$ & $121.6(10)$ & $\mathrm{C}(216)-\mathrm{C}(211)-\mathrm{P}(2)$ & $122.9(7)$ \\
\hline$C(116)-C(111)-C(112)$ & $116.4(11)$ & $\mathrm{C}(212)-\mathrm{C}(211)-\mathrm{P}(2)$ & $120.7(7)$ \\
\hline $\mathrm{C}(113)-\mathrm{C}(112)-\mathrm{C}(111)$ & $122.9(14)$ & $\mathrm{C}(211)-\mathrm{C}(212)-\mathrm{C}(213)$ & $120.7(10)$ \\
\hline $\mathrm{C}(114)-\mathrm{C}(113)-\mathrm{C}(112)$ & $119.7(16)$ & $\mathrm{C}(214)-\mathrm{C}(213)-\mathrm{C}(212)$ & $120.3(11)$ \\
\hline$C(113)-C(114)-C(115)$ & $121.0(15)$ & $\mathrm{C}(215)-\mathrm{C}(214)-\mathrm{C}(213)$ & $120.5(12)$ \\
\hline$C(116)-C(115)-C(114)$ & $117.9(14)$ & $\mathrm{C}(214)-\mathrm{C}(215)-\mathrm{C}(216)$ & $119.6(11)$ \\
\hline$C(111)-C(116)-C(115)$ & $122.0(11)$ & $\mathrm{C}(211)-\mathrm{C}(216)-\mathrm{C}(215)$ & $122.5(10)$ \\
\hline
\end{tabular}




\begin{tabular}{|c|c|c|c|}
\hline$C(122)-C(121)-C(126)$ & 120.0 & $\mathrm{C}(222)-\mathrm{C}(221)-\mathrm{C}(226)$ & $118.7(9)$ \\
\hline $\mathrm{C}(122)-\mathrm{C}(121)-\mathrm{P}(1)$ & $117.6(5)$ & $\mathrm{C}(222)-\mathrm{C}(221)-\mathrm{P}(2)$ & $123.8(7)$ \\
\hline $\mathrm{C}(126)-\mathrm{C}(121)-\mathrm{P}(1)$ & $122.4(5)$ & $\mathrm{C}(226)-\mathrm{C}(221)-\mathrm{P}(2)$ & $117.4(8)$ \\
\hline$C(123)-C(122)-C(121)$ & 120.0 & $\mathrm{C}(221)-\mathrm{C}(222)-\mathrm{C}(223)$ & $122.1(10)$ \\
\hline$C(122)-C(123)-C(124)$ & 120.0 & $C(224)-C(223)-C(222)$ & $118.9(11)$ \\
\hline$C(125)-C(124)-C(123)$ & 120.0 & $\mathrm{C}(223)-\mathrm{C}(224)-\mathrm{C}(225)$ & $120.5(12)$ \\
\hline$C(126)-C(125)-C(124)$ & 120.0 & $\mathrm{C}(224)-\mathrm{C}(225)-\mathrm{C}(226)$ & $120.5(13)$ \\
\hline$C(125)-C(126)-C(121)$ & 120.0 & $\mathrm{C}(221)-\mathrm{C}(226)-\mathrm{C}(225)$ & $119.1(12)$ \\
\hline$C(132)-C(131)-C(136)$ & $117.5(11)$ & $\mathrm{C}(236)-\mathrm{C}(231)-\mathrm{C}(232)$ & $118.6(9)$ \\
\hline $\mathrm{C}(132)-\mathrm{C}(131)-\mathrm{P}(1)$ & $121.4(9)$ & $\mathrm{C}(236)-\mathrm{C}(231)-\mathrm{P}(2)$ & $125.0(8)$ \\
\hline $\mathrm{C}(136)-\mathrm{C}(131)-\mathrm{P}(1)$ & $121.0(9)$ & $\mathrm{C}(232)-\mathrm{C}(231)-\mathrm{P}(2)$ & $116.4(7)$ \\
\hline$C(131)-C(132)-C(133)$ & $122.2(15)$ & $\mathrm{C}(233)-\mathrm{C}(232)-\mathrm{C}(231)$ & $120.6(10)$ \\
\hline $\mathrm{C}(134)-\mathrm{C}(133)-\mathrm{C}(132)$ & $118.9(19)$ & $\mathrm{C}(234)-\mathrm{C}(233)-\mathrm{C}(232)$ & $119.9(11)$ \\
\hline$C(135)-C(134)-C(133)$ & $120.1(19)$ & $\mathrm{C}(233)-\mathrm{C}(234)-\mathrm{C}(235)$ & $120.7(11)$ \\
\hline$C(134)-C(135)-C(136)$ & $118.9(17)$ & $\mathrm{C}(234)-\mathrm{C}(235)-\mathrm{C}(236)$ & $118.1(12)$ \\
\hline$C(135)-C(136)-C(131)$ & $122.0(14)$ & $C(231)-C(236)-C(235)$ & $122.1(11)$ \\
\hline$C(316)-C(311)-C(312)$ & $117.2(8)$ & $\mathrm{C}(416)-\mathrm{C}(411)-\mathrm{C}(412)$ & $118.3(9)$ \\
\hline C(316)-C(311)-P(3) & $122.4(7)$ & $\mathrm{C}(416)-\mathrm{C}(411)-\mathrm{P}(4)$ & $115.8(7)$ \\
\hline $\mathrm{C}(312)-\mathrm{C}(311)-\mathrm{P}(3)$ & 120.1(7) & $\mathrm{C}(412)-\mathrm{C}(411)-\mathrm{P}(4)$ & $125.7(8)$ \\
\hline $\mathrm{C}(313)-\mathrm{C}(312)-\mathrm{C}(311)$ & $120.9(9)$ & $\mathrm{C}(413)-\mathrm{C}(412)-\mathrm{C}(411)$ & $121.7(13)$ \\
\hline$C(314)-C(313)-C(312)$ & $119.8(10)$ & $C(412)-C(413)-C(414)$ & $120.7(15)$ \\
\hline$C(315)-C(314)-C(313)$ & $120.6(10)$ & $\mathrm{C}(413)-\mathrm{C}(414)-\mathrm{C}(415)$ & $120.1(14)$ \\
\hline$C(314)-C(315)-C(316)$ & $120.5(10)$ & $C(414)-C(415)-C(416)$ & $119.2(14)$ \\
\hline$C(311)-C(316)-C(315)$ & 121.1(9) & $C(411)-C(416)-C(415)$ & $119.9(12)$ \\
\hline$C(326)-C(321)-C(322)$ & $118.0(9)$ & $\mathrm{C}(422)-\mathrm{C}(421)-\mathrm{C}(426)$ & $116.2(8)$ \\
\hline $\mathrm{C}(326)-\mathrm{C}(321)-\mathrm{P}(3)$ & $119.7(8)$ & $\mathrm{C}(422)-\mathrm{C}(421)-\mathrm{P}(4)$ & $122.1(7)$ \\
\hline $\mathrm{C}(322)-\mathrm{C}(321)-\mathrm{P}(3)$ & $122.0(7)$ & $\mathrm{C}(426)-\mathrm{C}(421)-\mathrm{P}(4)$ & $121.6(7)$ \\
\hline $\mathrm{C}(321)-\mathrm{C}(322)-\mathrm{C}(323)$ & $121.9(10)$ & $\mathrm{C}(421)-\mathrm{C}(422)-\mathrm{C}(423)$ & $122.5(10)$ \\
\hline $\mathrm{C}(324)-\mathrm{C}(323)-\mathrm{C}(322)$ & $121.7(12)$ & $\mathrm{C}(424)-\mathrm{C}(423)-\mathrm{C}(422)$ & $120.3(10)$ \\
\hline $\mathrm{C}(325)-\mathrm{C}(324)-\mathrm{C}(323)$ & $116.8(12)$ & $\mathrm{C}(423)-\mathrm{C}(424)-\mathrm{C}(425)$ & $119.6(10)$ \\
\hline$C(324)-C(325)-C(326)$ & $122.3(12)$ & $\mathrm{C}(424)-\mathrm{C}(425)-\mathrm{C}(426)$ & $120.1(10)$ \\
\hline $\mathrm{C}(321)-\mathrm{C}(326)-\mathrm{C}(325)$ & $119.2(12)$ & $\mathrm{C}(421)-\mathrm{C}(426)-\mathrm{C}(425)$ & $121.1(9)$ \\
\hline$C(336)-C(331)-C(332)$ & $118.7(8)$ & $\mathrm{C}(432)-\mathrm{C}(431)-\mathrm{C}(436)$ & $117.5(9)$ \\
\hline $\mathrm{C}(336)-\mathrm{C}(331)-\mathrm{P}(3)$ & $125.5(7)$ & $\mathrm{C}(432)-\mathrm{C}(431)-\mathrm{P}(4)$ & $123.6(8)$ \\
\hline $\mathrm{C}(332)-\mathrm{C}(331)-\mathrm{P}(3)$ & $115.7(6)$ & $\mathrm{C}(436)-\mathrm{C}(431)-\mathrm{P}(4)$ & $118.5(7)$ \\
\hline $\mathrm{C}(333)-\mathrm{C}(332)-\mathrm{C}(331)$ & $120.0(9)$ & $\mathrm{C}(431)-\mathrm{C}(432)-\mathrm{C}(433)$ & $122.1(11)$ \\
\hline $\mathrm{C}(334)-\mathrm{C}(333)-\mathrm{C}(332)$ & $120.0(10)$ & $\mathrm{C}(434)-\mathrm{C}(433)-\mathrm{C}(432)$ & $121.6(12)$ \\
\hline $\mathrm{C}(335)-\mathrm{C}(334)-\mathrm{C}(333)$ & $120.6(10)$ & $\mathrm{C}(433)-\mathrm{C}(434)-\mathrm{C}(435)$ & $118.5(12)$ \\
\hline
\end{tabular}




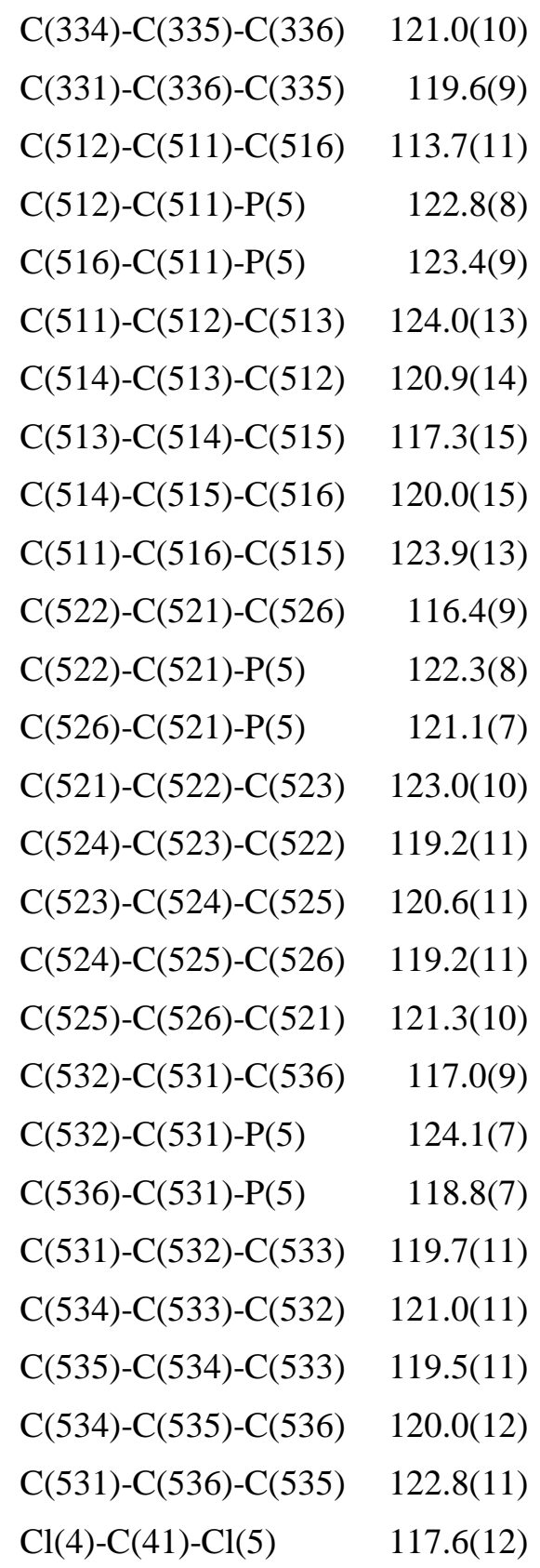

$\begin{array}{lr}\mathrm{C}(434)-\mathrm{C}(435)-\mathrm{C}(436) & 120.4(12) \\ \mathrm{C}(431)-\mathrm{C}(436)-\mathrm{C}(435) & 119.5(10) \\ \mathrm{C}(612)-\mathrm{C}(611)-\mathrm{C}(616) & 116.7(10) \\ \mathrm{C}(612)-\mathrm{C}(611)-\mathrm{P}(6) & 121.9(9) \\ \mathrm{C}(616)-\mathrm{C}(611)-\mathrm{P}(6) & 121.2(9) \\ \mathrm{C}(611)-\mathrm{C}(612)-\mathrm{C}(613) & 122.2(13) \\ \mathrm{C}(614)-\mathrm{C}(613)-\mathrm{C}(612) & 120.8(15) \\ \mathrm{C}(613)-\mathrm{C}(614)-\mathrm{C}(615) & 120.0(15) \\ \mathrm{C}(614)-\mathrm{C}(615)-\mathrm{C}(616) & 120.2(14) \\ \mathrm{C}(611)-\mathrm{C}(616)-\mathrm{C}(615) & 120.2(12) \\ \mathrm{C}(622)-\mathrm{C}(621)-\mathrm{C}(626) & 116.2(13) \\ \mathrm{C}(622)-\mathrm{C}(621)-\mathrm{P}(6) & 125.0(8) \\ \mathrm{C}(626)-\mathrm{C}(621)-\mathrm{P}(6) & 118.2(12) \\ \mathrm{C}(621)-\mathrm{C}(622)-\mathrm{C}(623) & 124.5(13) \\ \mathrm{C}(624)-\mathrm{C}(623)-\mathrm{C}(622) & 117.9(15) \\ \mathrm{C}(623)-\mathrm{C}(624)-\mathrm{C}(625) & 118.6(19) \\ \mathrm{C}(626)-\mathrm{C}(625)-\mathrm{C}(624) & 121(2) \\ \mathrm{C}(625)-\mathrm{C}(626)-\mathrm{C}(621) & 121.0(18) \\ \mathrm{C}(636)-\mathrm{C}(631)-\mathrm{C}(632) & 116.6(11) \\ \mathrm{C}(636)-\mathrm{C}(631)-\mathrm{P}(6) & 124.0(10) \\ \mathrm{C}(632)-\mathrm{C}(631)-\mathrm{P}(6) & 119.3(8) \\ \mathrm{C}(631)-\mathrm{C}(632)-\mathrm{C}(633) & 121.0(12) \\ \mathrm{C}(634)-\mathrm{C}(633)-\mathrm{C}(632) & 120.8(14) \\ \mathrm{C}(635)-\mathrm{C}(634)-\mathrm{C}(633) & 118.4(15) \\ \mathrm{C}(634)-\mathrm{C}(635)-\mathrm{C}(636) & 119.7(16) \\ \mathrm{C}(631)-\mathrm{C}(636)-\mathrm{C}(635) & 123.3(14) \\ \mathrm{Cl}(7)-\mathrm{C}(42)-\mathrm{Cl}(6) & 115.6(16) \\ & \end{array}$


Table S4. Anisotropic displacement parameters $\left(\AA^{2} \times 10^{3}\right)$ for 1,3,5-

$\left[\mathrm{Cl}(\mathrm{CO})(\mathrm{Py})\left(\mathrm{PPh}_{3}\right)_{2} \mathrm{RuCH}=\mathrm{CH}\right]_{3} \mathrm{C}_{6} \mathrm{H}_{3} \cdot \mathrm{CH}_{2} \mathrm{Cl}_{2}\left(\mathbf{5} \cdot \mathrm{CH}_{2} \mathrm{Cl}_{2}\right)$.

\begin{tabular}{|c|c|c|c|c|c|c|}
\hline Atom & $U 11$ & $U 22$ & U33 & $U 23$ & $U 13$ & U12 \\
\hline $\mathrm{Ru}(1)$ & $61(1)$ & $44(1)$ & $45(1)$ & $-1(1)$ & $13(1)$ & 10(1) \\
\hline $\mathrm{Ru}(2)$ & $42(1)$ & $47(1)$ & $44(1)$ & $2(1)$ & $10(1)$ & 1(1) \\
\hline $\mathrm{Ru}(3)$ & $50(1)$ & $40(1)$ & $51(1)$ & $2(1)$ & $16(1)$ & $8(1)$ \\
\hline $\mathrm{Cl}(1)$ & $104(2)$ & $63(1)$ & $65(2)$ & $8(1)$ & $28(1)$ & $12(1)$ \\
\hline $\mathrm{Cl}(2)$ & $52(1)$ & $67(1)$ & $72(2)$ & $0(1)$ & $17(1)$ & 12(1) \\
\hline $\mathrm{Cl}(3)$ & $78(2)$ & $63(1)$ & $60(1)$ & $-5(1)$ & $14(1)$ & $-9(1)$ \\
\hline $\mathrm{P}(1)$ & $68(2)$ & $55(1)$ & $60(2)$ & $-6(1)$ & $0(1)$ & 11(1) \\
\hline $\mathrm{P}(2)$ & $57(1)$ & $56(1)$ & $54(1)$ & $0(1)$ & $19(1)$ & $3(1)$ \\
\hline $\mathrm{P}(3)$ & $50(1)$ & $52(1)$ & $53(1)$ & $-7(1)$ & $13(1)$ & $-6(1)$ \\
\hline $\mathrm{P}(4)$ & $48(1)$ & $49(1)$ & $59(1)$ & $5(1)$ & $17(1)$ & $-1(1)$ \\
\hline $\mathrm{P}(5)$ & $47(1)$ & $56(1)$ & $56(1)$ & $2(1)$ & $17(1)$ & $9(1)$ \\
\hline$P(6)$ & $64(2)$ & $66(2)$ & $63(2)$ & $-7(1)$ & $26(1)$ & $-13(1)$ \\
\hline $\mathrm{C}(1)$ & $68(6)$ & $60(6)$ & $67(6)$ & $4(5)$ & $12(5)$ & $4(5)$ \\
\hline$C(2)$ & $47(5)$ & $54(5)$ & $51(5)$ & $-3(4)$ & $10(4)$ & $-3(4)$ \\
\hline$C(3)$ & $46(5)$ & $60(5)$ & $77(7)$ & $13(5)$ & $23(5)$ & $12(4)$ \\
\hline $\mathrm{O}(1)$ & $85(5)$ & $63(4)$ & $141(7)$ & $31(5)$ & $10(5)$ & $3(4)$ \\
\hline $\mathrm{O}(2)$ & $80(5)$ & $74(4)$ & $96(5)$ & $-13(4)$ & $43(4)$ & $10(4)$ \\
\hline $\mathrm{O}(3)$ & $64(4)$ & $124(6)$ & $58(4)$ & $-6(4)$ & $-1(3)$ & $1(4)$ \\
\hline $\mathrm{N}(1)$ & $84(5)$ & $46(4)$ & $64(5)$ & $-13(4)$ & $16(4)$ & $13(4)$ \\
\hline $\mathrm{N}(2)$ & $51(4)$ & $95(6)$ & $55(5)$ & $13(4)$ & $10(4)$ & $0(4)$ \\
\hline $\mathrm{N}(3)$ & $92(6)$ & $50(5)$ & $86(6)$ & $7(4)$ & $34(5)$ & $18(4)$ \\
\hline $\mathrm{C}(01)$ & $41(4)$ & $31(4)$ & $52(5)$ & $-2(3)$ & $13(4)$ & $0(3)$ \\
\hline $\mathrm{C}(02)$ & $45(4)$ & $39(4)$ & $52(5)$ & $3(4)$ & $12(4)$ & $6(3)$ \\
\hline $\mathrm{C}(03)$ & $51(5)$ & $38(4)$ & $52(5)$ & $3(4)$ & $20(4)$ & $3(4)$ \\
\hline $\mathrm{C}(04)$ & $48(5)$ & $40(4)$ & $49(5)$ & $-9(4)$ & $15(4)$ & $6(4)$ \\
\hline $\mathrm{C}(05)$ & $44(4)$ & $44(4)$ & $45(5)$ & $5(4)$ & $10(4)$ & $8(3)$ \\
\hline$C(06)$ & $50(5)$ & $48(5)$ & $39(4)$ & $3(4)$ & $9(4)$ & $5(4)$ \\
\hline$C(11)$ & $45(5)$ & $49(5)$ & $48(5)$ & $0(4)$ & $-1(4)$ & $8(4)$ \\
\hline$C(12)$ & $63(5)$ & $43(5)$ & $46(5)$ & $5(4)$ & $11(4)$ & $10(4)$ \\
\hline $\mathrm{C}(21)$ & $48(5)$ & $42(4)$ & $47(5)$ & $-3(4)$ & $13(4)$ & $-9(4)$ \\
\hline$C(22)$ & $47(5)$ & $39(4)$ & $46(5)$ & $2(4)$ & $14(4)$ & $3(4)$ \\
\hline$C(31)$ & $45(5)$ & $46(5)$ & $55(5)$ & $6(4)$ & $18(4)$ & $10(4)$ \\
\hline$C(32)$ & $55(5)$ & $54(5)$ & $42(5)$ & $5(4)$ & $12(4)$ & $16(4)$ \\
\hline $\mathrm{C}(101)$ & $129(9)$ & $69(6)$ & $61(7)$ & $3(5)$ & $33(6)$ & $14(6)$ \\
\hline
\end{tabular}




\begin{tabular}{|c|c|c|c|c|c|c|}
\hline$C(102)$ & $141(11)$ & $91(8)$ & $89(8)$ & $-17(7)$ & $63(8)$ & $8(8)$ \\
\hline$C(103)$ & 121(10) & 111(10) & $92(10)$ & $-34(8)$ & $47(8)$ & $11(8)$ \\
\hline C(104) & 114(9) & $75(7)$ & 100(9) & $-24(7)$ & $19(8)$ & $32(6)$ \\
\hline$C(105)$ & $97(8)$ & $61(6)$ & $69(7)$ & $-20(5)$ & $8(6)$ & $13(5)$ \\
\hline$C(201)$ & $49(5)$ & 124(9) & $70(7)$ & $27(6)$ & $5(5)$ & $14(6)$ \\
\hline$C(202)$ & $76(8)$ & $184(14)$ & $81(9)$ & $43(9)$ & $18(7)$ & $44(8)$ \\
\hline$C(203)$ & $92(10)$ & $270(20)$ & $76(10)$ & $37(12)$ & $-5(8)$ & $65(12)$ \\
\hline$C(204)$ & $105(10)$ & $249(18)$ & $52(7)$ & $-28(9)$ & $30(7)$ & $23(11)$ \\
\hline$C(205)$ & $80(7)$ & 132(9) & $57(7)$ & $-14(6)$ & $20(6)$ & $14(6)$ \\
\hline $\mathrm{C}(301)$ & $143(11)$ & $57(7)$ & $120(10)$ & $-7(7)$ & $35(8)$ & $25(7)$ \\
\hline$C(302)$ & $217(17)$ & $81(10)$ & $168(16)$ & $-23(10)$ & $82(14)$ & $46(10)$ \\
\hline$C(303)$ & $410(30)$ & $56(9)$ & $160(17)$ & $14(10)$ & $110(19)$ & $48(13)$ \\
\hline$C(304)$ & $218(17)$ & $75(9)$ & $125(12)$ & $23(9)$ & $40(12)$ & $33(9)$ \\
\hline$C(305)$ & 113(9) & $59(7)$ & 101(9) & $17(6)$ & $9(7)$ & $20(6)$ \\
\hline$C(111)$ & $67(7)$ & $63(6)$ & $88(8)$ & $-16(6)$ & $-4(6)$ & $5(5)$ \\
\hline$C(112)$ & $88(9)$ & $138(11)$ & $108(10)$ & $-17(9)$ & $18(8)$ & $-13(8)$ \\
\hline $\mathrm{C}(113)$ & $109(12)$ & $188(17)$ & $151(16)$ & $-43(14)$ & $45(12)$ & $-41(11)$ \\
\hline $\mathrm{C}(114)$ & $98(11)$ & $107(10)$ & $240(20)$ & $-2(13)$ & $88(14)$ & $-21(8)$ \\
\hline$C(115)$ & $80(9)$ & $119(10)$ & $164(13)$ & $6(9)$ & $51(9)$ & $-17(8)$ \\
\hline$C(116)$ & $62(7)$ & $89(8)$ & 111(10) & $-12(7)$ & $18(7)$ & $-9(6)$ \\
\hline $\mathrm{C}(121)$ & 110(9) & $81(7)$ & $59(6)$ & $-14(5)$ & $-15(6)$ & $-3(6)$ \\
\hline$C(122)$ & 112(9) & $93(8)$ & $83(8)$ & $-29(7)$ & $14(7)$ & $-3(7)$ \\
\hline$C(123)$ & $195(16)$ & $130(12)$ & $113(12)$ & $-69(10)$ & $28(11)$ & $-9(11)$ \\
\hline $\mathrm{C}(124)$ & $470(40)$ & $200(20)$ & $70(12)$ & $-21(13)$ & $7(18)$ & $10(20)$ \\
\hline$C(125)$ & $670(60)$ & $156(17)$ & 71(12) & $-24(11)$ & $-120(20)$ & $100(30)$ \\
\hline$C(126)$ & $340(30)$ & $138(13)$ & $67(9)$ & $-8(9)$ & $-87(13)$ & $47(14)$ \\
\hline $\mathrm{C}(131)$ & $89(8)$ & $61(7)$ & $90(8)$ & $-4(6)$ & $-20(6)$ & $20(6)$ \\
\hline$C(132)$ & $200(16)$ & $138(13)$ & 134(13) & $28(10)$ & $59(12)$ & $108(12)$ \\
\hline C(133) & $360(30)$ & $200(20)$ & $200(20)$ & $2(18)$ & $60(20)$ & $200(30)$ \\
\hline C(134) & $200(20)$ & $115(15)$ & $240(30)$ & $-44(15)$ & $-31(19)$ & $96(16)$ \\
\hline$C(135)$ & $149(16)$ & $75(11)$ & $230(20)$ & $23(11)$ & $-37(14)$ & $12(10)$ \\
\hline$C(136)$ & $97(9)$ & $62(7)$ & $173(14)$ & $10(8)$ & $-15(9)$ & $20(7)$ \\
\hline $\mathrm{C}(211)$ & $53(5)$ & $67(6)$ & $57(6)$ & $5(5)$ & $8(4)$ & $8(4)$ \\
\hline$C(212)$ & $66(6)$ & $74(7)$ & $75(7)$ & $19(5)$ & $17(5)$ & $14(5)$ \\
\hline$C(213)$ & $80(7)$ & $84(8)$ & 93(9) & $23(7)$ & $21(7)$ & $23(6)$ \\
\hline$C(214)$ & $82(8)$ & $126(11)$ & $87(9)$ & $32(8)$ & $20(7)$ & $19(8)$ \\
\hline$C(215)$ & $98(9)$ & $140(11)$ & $57(7)$ & $-4(7)$ & $23(6)$ & $8(8)$ \\
\hline$C(216)$ & $76(7)$ & $86(7)$ & $62(7)$ & $0(6)$ & $10(5)$ & $2(5)$ \\
\hline
\end{tabular}




\begin{tabular}{|c|c|c|c|c|c|c|}
\hline$C(221)$ & $61(6)$ & $68(6)$ & $65(6)$ & $-7(5)$ & $18(5)$ & $-17(5)$ \\
\hline$C(222)$ & $79(7)$ & $69(7)$ & 109(9) & $-4(6)$ & $19(6)$ & $-3(6)$ \\
\hline$C(223)$ & $94(9)$ & $68(7)$ & $150(12)$ & $-19(7)$ & $24(8)$ & $-11(6)$ \\
\hline$C(224)$ & $128(12)$ & $92(10)$ & $144(12)$ & $-34(8)$ & $54(10)$ & $-37(9)$ \\
\hline$C(225)$ & $91(10)$ & 161(14) & $159(14)$ & $-61(11)$ & $43(9)$ & $-46(10)$ \\
\hline$C(226)$ & $94(9)$ & $110(9)$ & $124(11)$ & $-45(8)$ & $31(8)$ & $-29(7)$ \\
\hline$C(231)$ & $55(5)$ & $65(6)$ & $73(6)$ & $3(5)$ & $22(5)$ & $-1(5)$ \\
\hline$C(232)$ & $78(7)$ & $77(7)$ & $89(8)$ & $3(6)$ & $38(6)$ & $0(5)$ \\
\hline$C(233)$ & $102(9)$ & 108(9) & 103(9) & $-6(7)$ & $66(8)$ & $-7(7)$ \\
\hline$C(234)$ & $87(9)$ & $150(12)$ & $113(11)$ & $-14(9)$ & $58(8)$ & $12(8)$ \\
\hline$C(235)$ & $89(9)$ & $153(12)$ & 149(13) & $22(10)$ & $56(9)$ & $49(8)$ \\
\hline$C(236)$ & $76(7)$ & $129(10)$ & 104(9) & $38(7)$ & $41(7)$ & $27(7)$ \\
\hline$C(311)$ & $60(5)$ & $48(5)$ & $54(5)$ & $-7(4)$ & $10(4)$ & $-16(4)$ \\
\hline$C(312)$ & $93(7)$ & $58(6)$ & $65(6)$ & $-10(5)$ & $24(6)$ & $2(5)$ \\
\hline$C(313)$ & $83(7)$ & $80(7)$ & $70(7)$ & $-19(6)$ & $12(6)$ & $9(6)$ \\
\hline$C(314)$ & $74(7)$ & $69(7)$ & $87(8)$ & $-7(6)$ & $9(6)$ & $1(5)$ \\
\hline$C(315)$ & $71(7)$ & $80(7)$ & $72(7)$ & $24(6)$ & $9(6)$ & $-5(6)$ \\
\hline$C(316)$ & $59(6)$ & $65(6)$ & $69(6)$ & $-1(5)$ & $15(5)$ & $-11(5)$ \\
\hline$C(321)$ & $55(5)$ & $63(5)$ & $63(6)$ & $-5(4)$ & $19(5)$ & $-15(4)$ \\
\hline$C(322)$ & $127(10)$ & $123(9)$ & $102(9)$ & $-59(7)$ & $80(8)$ & $-74(8)$ \\
\hline$C(323)$ & $138(12)$ & $154(12)$ & $120(11)$ & $-67(9)$ & $79(10)$ & $-88(10)$ \\
\hline$C(324)$ & $125(12)$ & 173(13) & $108(11)$ & $-25(10)$ & $80(10)$ & $-28(10)$ \\
\hline$C(325)$ & $105(11)$ & $280(20)$ & $120(13)$ & $-44(13)$ & $53(10)$ & $-95(12)$ \\
\hline$C(326)$ & $110(10)$ & $245(17)$ & $96(10)$ & $-49(10)$ & $52(8)$ & $-97(11)$ \\
\hline $\mathrm{C}(331)$ & $54(5)$ & $62(5)$ & $49(5)$ & $-8(4)$ & 11(4) & $-7(4)$ \\
\hline$C(332)$ & $62(6)$ & $69(6)$ & $74(7)$ & $-14(5)$ & $4(5)$ & $-1(5)$ \\
\hline$C(333)$ & $77(7)$ & $72(7)$ & 111(9) & $-14(7)$ & $-17(7)$ & $8(6)$ \\
\hline$C(334)$ & $88(8)$ & 93(9) & $83(8)$ & $-5(7)$ & $-22(6)$ & $-4(7)$ \\
\hline$C(335)$ & $123(10)$ & $77(7)$ & $76(8)$ & $-25(6)$ & $-15(7)$ & $5(7)$ \\
\hline$C(336)$ & $85(7)$ & $64(6)$ & $76(7)$ & $-15(5)$ & $-7(6)$ & $-5(5)$ \\
\hline $\mathrm{C}(411)$ & $48(5)$ & $62(6)$ & $64(6)$ & $9(4)$ & $17(4)$ & $-5(4)$ \\
\hline$C(412)$ & $152(11)$ & $57(7)$ & 108(9) & $31(6)$ & $45(8)$ & $25(7)$ \\
\hline$C(413)$ & 213(18) & $85(10)$ & $173(17)$ & $50(11)$ & $70(15)$ & $41(10)$ \\
\hline$C(414)$ & $187(16)$ & $114(13)$ & $162(17)$ & $81(13)$ & $60(14)$ & $34(11)$ \\
\hline$C(415)$ & $144(12)$ & $155(14)$ & $96(10)$ & $57(10)$ & 40(9) & $20(10)$ \\
\hline$C(416)$ & $85(7)$ & $99(8)$ & $80(8)$ & $20(7)$ & $26(6)$ & $4(6)$ \\
\hline $\mathrm{C}(421)$ & $50(5)$ & $58(5)$ & $55(5)$ & $-4(4)$ & $16(4)$ & $-1(4)$ \\
\hline$C(422)$ & $52(6)$ & $88(7)$ & $110(9)$ & $29(6)$ & $18(6)$ & $3(5)$ \\
\hline
\end{tabular}




\begin{tabular}{|c|c|c|c|c|c|c|}
\hline$C(423)$ & $55(7)$ & 105(9) & $120(10)$ & $24(7)$ & $17(6)$ & $-15(6)$ \\
\hline$C(424)$ & $50(6)$ & $100(8)$ & 106(9) & $-1(7)$ & $26(6)$ & $-9(6)$ \\
\hline$C(425)$ & $47(6)$ & $79(7)$ & 121(9) & $7(6)$ & $4(6)$ & $6(5)$ \\
\hline$C(426)$ & $48(5)$ & $71(6)$ & 103(8) & $12(6)$ & $18(5)$ & $1(5)$ \\
\hline$C(431)$ & $51(5)$ & $63(6)$ & $68(6)$ & $-9(5)$ & $14(5)$ & $-6(4)$ \\
\hline$C(432)$ & $70(7)$ & $88(7)$ & 112(9) & $-27(7)$ & $37(6)$ & $-11(6)$ \\
\hline$C(433)$ & $97(9)$ & $94(9)$ & $134(12)$ & $-45(9)$ & $53(9)$ & $-17(7)$ \\
\hline$C(434)$ & $130(12)$ & 121(11) & $104(11)$ & $-43(9)$ & $72(10)$ & $-33(9)$ \\
\hline$C(435)$ & 104(9) & 113(9) & $75(8)$ & $-13(7)$ & $24(7)$ & $-39(8)$ \\
\hline$C(436)$ & $80(7)$ & $75(6)$ & $67(7)$ & $-17(5)$ & $26(6)$ & $-21(5)$ \\
\hline$C(511)$ & $57(6)$ & $76(6)$ & $63(6)$ & $14(5)$ & $24(5)$ & $11(5)$ \\
\hline$C(512)$ & $106(10)$ & $225(16)$ & $77(9)$ & $76(10)$ & $-1(8)$ & $-53(10)$ \\
\hline $\mathrm{C}(513)$ & $81(9)$ & $290(20)$ & $114(12)$ & $95(14)$ & $-8(8)$ & $-53(12)$ \\
\hline$C(514)$ & $121(12)$ & $144(14)$ & $108(12)$ & $48(11)$ & $27(10)$ & $71(10)$ \\
\hline $\mathrm{C}(515)$ & $430(40)$ & $78(10)$ & $127(15)$ & $42(11)$ & $50(19)$ & $14(15)$ \\
\hline$C(516)$ & $254(19)$ & $86(10)$ & $102(11)$ & $30(8)$ & $21(11)$ & $5(11)$ \\
\hline$C(521)$ & $59(6)$ & $54(5)$ & $75(6)$ & $2(5)$ & $28(5)$ & $-2(4)$ \\
\hline$C(522)$ & $78(8)$ & 107(9) & $152(12)$ & $-53(9)$ & $51(8)$ & $-10(7)$ \\
\hline$C(523)$ & 89(9) & 118(10) & $175(14)$ & $-77(10)$ & $66(9)$ & $-34(8)$ \\
\hline$C(524)$ & 119(11) & $96(9)$ & $98(9)$ & $-33(7)$ & $15(8)$ & $-9(8)$ \\
\hline$C(525)$ & $63(7)$ & $86(8)$ & $153(12)$ & $-12(8)$ & $0(7)$ & $-7(6)$ \\
\hline$C(526)$ & $69(7)$ & $71(7)$ & $127(10)$ & $-16(6)$ & $24(7)$ & $0(6)$ \\
\hline$C(531)$ & $50(5)$ & $62(5)$ & $52(5)$ & $0(4)$ & $14(4)$ & $11(4)$ \\
\hline$C(532)$ & 118(9) & 105(9) & $78(8)$ & $-1(6)$ & $39(7)$ & $26(7)$ \\
\hline$C(533)$ & $170(14)$ & 131(11) & $95(10)$ & $-24(9)$ & $65(10)$ & $45(10)$ \\
\hline$C(534)$ & 97(9) & $85(8)$ & $148(13)$ & $5(9)$ & $65(9)$ & $36(7)$ \\
\hline$C(535)$ & $84(8)$ & $90(8)$ & $135(12)$ & $-7(8)$ & $30(8)$ & $34(6)$ \\
\hline$C(536)$ & $92(8)$ & $96(8)$ & $88(8)$ & $-5(6)$ & $29(6)$ & $31(7)$ \\
\hline$C(611)$ & $63(6)$ & $96(8)$ & $63(6)$ & $-11(6)$ & $32(5)$ & $-25(6)$ \\
\hline$C(612)$ & $129(11)$ & $117(10)$ & $72(8)$ & $9(7)$ & $38(8)$ & $-10(8)$ \\
\hline$C(613)$ & $133(12)$ & 171(15) & $65(9)$ & $-3(9)$ & $25(8)$ & $-16(11)$ \\
\hline$C(614)$ & $109(11)$ & $176(16)$ & $84(10)$ & $-38(11)$ & $39(9)$ & $-38(11)$ \\
\hline$C(615)$ & $109(11)$ & 139(12) & $115(12)$ & $-52(10)$ & $58(9)$ & $-30(9)$ \\
\hline$C(616)$ & $80(7)$ & 112(9) & $88(8)$ & $-32(7)$ & $33(6)$ & $-15(7)$ \\
\hline$C(621)$ & $45(6)$ & 118(9) & 100(9) & $-38(7)$ & $23(6)$ & $-10(6)$ \\
\hline$C(622)$ & $58(7)$ & $227(17)$ & $116(11)$ & $69(11)$ & $21(7)$ & $31(9)$ \\
\hline$C(623)$ & $94(11)$ & $330(20)$ & $112(12)$ & $43(13)$ & $37(9)$ & $92(14)$ \\
\hline$C(624)$ & $44(9)$ & $380(40)$ & $220(20)$ & $-160(20)$ & $-24(11)$ & $61(18)$ \\
\hline
\end{tabular}




\begin{tabular}{lrrrrrr}
$\mathrm{C}(625)$ & $79(16)$ & $260(30)$ & $570(60)$ & $-150(30)$ & $50(20)$ & $-25(18)$ \\
$\mathrm{C}(626)$ & $75(11)$ & $145(15)$ & $420(40)$ & $-56(17)$ & $37(16)$ & $-8(10)$ \\
$\mathrm{C}(631)$ & $136(10)$ & $79(7)$ & $80(8)$ & $-4(6)$ & $42(7)$ & $-47(7)$ \\
$\mathrm{C}(632)$ & $166(13)$ & $69(8)$ & $123(11)$ & $-9(7)$ & $50(10)$ & $-41(8)$ \\
$\mathrm{C}(633)$ & $232(18)$ & $82(10)$ & $143(14)$ & $-7(9)$ & $54(13)$ & $-50(10)$ \\
$\mathrm{C}(634)$ & $320(30)$ & $126(14)$ & $179(19)$ & $39(13)$ & $51(19)$ & $-124(16)$ \\
$\mathrm{C}(635)$ & $750(70)$ & $250(30)$ & $170(20)$ & $-67(18)$ & $200(30)$ & $-350(40)$ \\
$\mathrm{C}(636)$ & $420(30)$ & $202(18)$ & $140(14)$ & $-63(13)$ & $168(19)$ & $-200(20)$ \\
$\mathrm{C}(41)$ & $330(50)$ & $180(30)$ & $77(18)$ & $-51(19)$ & $-90(20)$ & $210(40)$ \\
$\mathrm{Cl}(4)$ & $350(16)$ & $118(7)$ & $160(9)$ & $-2(6)$ & $74(10)$ & $9(8)$ \\
$\mathrm{Cl}(5)$ & $136(7)$ & $309(13)$ & $125(7)$ & $36(8)$ & $22(6)$ & $49(8)$ \\
\hline \multicolumn{7}{l}{$\mathrm{The}$ anisotropic displacement factor exponent takes the form: $-2 \pi^{2}\left[\mathrm{~h}^{2} \mathrm{a}^{2} \mathrm{U} 11+\ldots+2 \mathrm{hka*b} \mathrm{U} 12\right]}$.
\end{tabular}


Table S5. Hydrogen coordinates ( $\left.\mathrm{x} 10^{4}\right)$ and isotropic displacement parameters $\left(\AA^{2} \times 10^{3}\right)$ for 1,3,5-[Cl(CO)(Py) $\left.\left(\mathrm{PPh}_{3}\right)_{2} \mathrm{RuCH}=\mathrm{CH}\right]_{3} \mathrm{C}_{6} \mathrm{H}_{3} \cdot \mathrm{CH}_{2} \mathrm{Cl}_{2}\left(\mathbf{5} \cdot \mathrm{CH}_{2} \mathrm{Cl}_{2}\right)$.

\begin{tabular}{|c|c|c|c|c|}
\hline Atom & $x$ & $y$ & $z$ & $U(e q)$ \\
\hline $\mathrm{H}(02 \mathrm{~A})$ & 7721 & 5771 & 2702 & 55 \\
\hline $\mathrm{H}(04 \mathrm{~A})$ & 7008 & 7636 & 2868 & 54 \\
\hline $\mathrm{H}(06 \mathrm{~A})$ & 6957 & 6960 & 1620 & 55 \\
\hline $\mathrm{H}(11 \mathrm{~A})$ & 7334 & 6177 & 1258 & 60 \\
\hline $\mathrm{H}(12 \mathrm{~A})$ & 7632 & 5285 & 1969 & 61 \\
\hline $\mathrm{H}(21 \mathrm{~A})$ & 8102 & 5832 & 3431 & 55 \\
\hline $\mathrm{H}(22 \mathrm{~A})$ & 7400 & 6918 & 3537 & 52 \\
\hline $\mathrm{H}(31 \mathrm{~A})$ & 6838 & 8601 & 2559 & 57 \\
\hline $\mathrm{H}(32 \mathrm{~A})$ & 6480 & 8099 & 1699 & 61 \\
\hline $\mathrm{H}(10 \mathrm{~A})$ & 7970 & 5222 & -49 & 102 \\
\hline $\mathrm{H}(10 \mathrm{~B})$ & 8381 & 4660 & -560 & 121 \\
\hline $\mathrm{H}(10 \mathrm{C})$ & 8891 & 3627 & -361 & 125 \\
\hline $\mathrm{H}(10 \mathrm{D})$ & 8939 & 3153 & 339 & 118 \\
\hline $\mathrm{H}(10 \mathrm{E})$ & 8479 & 3718 & 832 & 94 \\
\hline $\mathrm{H}(20 \mathrm{~A})$ & 9155 & 4817 & 4942 & 99 \\
\hline $\mathrm{H}(20 \mathrm{~B})$ & 9556 & 4537 & 5692 & 137 \\
\hline $\mathrm{H}(20 \mathrm{C})$ & 9463 & 5301 & 6249 & 181 \\
\hline $\mathrm{H}(20 \mathrm{D})$ & 8846 & 6269 & 6053 & 160 \\
\hline $\mathrm{H}(20 \mathrm{E})$ & 8416 & 6519 & 5279 & 107 \\
\hline $\mathrm{H}(30 \mathrm{~A})$ & 6104 & 10951 & 2713 & 128 \\
\hline $\mathrm{H}(30 \mathrm{~B})$ & 5885 & 12120 & 2658 & 179 \\
\hline $\mathrm{H}(30 \mathrm{C})$ & 5787 & 12705 & 1980 & 241 \\
\hline $\mathrm{H}(30 \mathrm{D})$ & 5697 & 12042 & 1329 & 169 \\
\hline $\mathrm{H}(30 \mathrm{E})$ & 5887 & 10879 & 1405 & 114 \\
\hline $\mathrm{H}(11 \mathrm{~B})$ & 5661 & 4257 & 152 & 135 \\
\hline $\mathrm{H}(11 \mathrm{C})$ & 5006 & 3596 & 421 & 177 \\
\hline $\mathrm{H}(11 \mathrm{D})$ & 5121 & 3513 & 1181 & 167 \\
\hline $\mathrm{H}(11 \mathrm{E})$ & 5964 & 4028 & 1698 & 141 \\
\hline $\mathrm{H}(11 \mathrm{~F})$ & 6644 & 4677 & 1414 & 106 \\
\hline $\mathrm{H}(12 \mathrm{~B})$ & 7082 & 3843 & 142 & 118 \\
\hline $\mathrm{H}(12 \mathrm{C})$ & 6899 & 3354 & -580 & 178 \\
\hline $\mathrm{H}(12 \mathrm{D})$ & 6304 & 3951 & -1208 & 310 \\
\hline $\mathrm{H}(12 \mathrm{E})$ & 5893 & 5036 & -1114 & 409 \\
\hline $\mathrm{H}(12 \mathrm{~F})$ & 6076 & 5525 & -392 & 251 \\
\hline
\end{tabular}




\begin{tabular}{|c|c|c|c|c|}
\hline $\mathrm{H}(13 \mathrm{~A})$ & 5847 & 5726 & 880 & 185 \\
\hline $\mathrm{H}(13 \mathrm{~B})$ & 5394 & 6815 & 843 & 305 \\
\hline $\mathrm{H}(13 \mathrm{C})$ & 5739 & 7713 & 466 & 245 \\
\hline $\mathrm{H}(13 \mathrm{D})$ & 6364 & 7455 & 3 & 203 \\
\hline $\mathrm{H}(13 \mathrm{E})$ & 6808 & 6363 & 49 & 145 \\
\hline $\mathrm{H}(21 \mathrm{~B})$ & 8914 & 3878 & 1736 & 87 \\
\hline $\mathrm{H}(21 \mathrm{C})$ & 9184 & 3316 & 2435 & 103 \\
\hline $\mathrm{H}(21 \mathrm{D})$ & 9340 & 3943 & 3089 & 118 \\
\hline $\mathrm{H}(21 \mathrm{E})$ & 9263 & 5129 & 3066 & 118 \\
\hline $\mathrm{H}(21 \mathrm{~F})$ & 9059 & 5704 & 2377 & 92 \\
\hline $\mathrm{H}(22 \mathrm{~B})$ & 8288 & 6695 & 1502 & 104 \\
\hline $\mathrm{H}(22 \mathrm{C})$ & 8659 & 7798 & 1735 & 127 \\
\hline $\mathrm{H}(22 \mathrm{D})$ & 9691 & 7971 & 1978 & 141 \\
\hline $\mathrm{H}(22 \mathrm{E})$ & 10353 & 7051 & 2005 & 162 \\
\hline $\mathrm{H}(22 \mathrm{~F})$ & 9981 & 5938 & 1776 & 131 \\
\hline $\mathrm{H}(23 \mathrm{~A})$ & 9174 & 5723 & 656 & 94 \\
\hline $\mathrm{H}(23 \mathrm{~B})$ & 9909 & 5419 & 291 & 116 \\
\hline $\mathrm{H}(23 \mathrm{C})$ & 10601 & 4545 & 584 & 133 \\
\hline $\mathrm{H}(23 \mathrm{D})$ & 10552 & 3943 & 1236 & 151 \\
\hline $\mathrm{H}(23 \mathrm{E})$ & 9830 & 4288 & 1610 & 120 \\
\hline $\mathrm{H}(31 \mathrm{~B})$ & 8265 & 7848 & 4740 & 86 \\
\hline $\mathrm{H}(31 \mathrm{C})$ & 7675 & 8839 & 4542 & 95 \\
\hline $\mathrm{H}(31 \mathrm{D})$ & 7575 & 9353 & 3844 & 95 \\
\hline $\mathrm{H}(31 \mathrm{E})$ & 8042 & 8892 & 3349 & 92 \\
\hline $\mathrm{H}(31 \mathrm{~F})$ & 8649 & 7910 & 3540 & 77 \\
\hline $\mathrm{H}(32 \mathrm{~B})$ & 8877 & 6496 & 3387 & 130 \\
\hline $\mathrm{H}(32 \mathrm{C})$ & 9442 & 6637 & 2881 & 155 \\
\hline $\mathrm{H}(32 \mathrm{D})$ & 10410 & 7087 & 3104 & 151 \\
\hline $\mathrm{H}(32 \mathrm{E})$ & 10739 & 7526 & 3822 & 196 \\
\hline $\mathrm{H}(32 \mathrm{~F})$ & 10153 & 7426 & 4340 & 175 \\
\hline $\mathrm{H}(33 \mathrm{~A})$ & 9867 & 6120 & 4872 & 85 \\
\hline $\mathrm{H}(33 \mathrm{~B})$ & 10572 & 6218 & 5577 & 114 \\
\hline $\mathrm{H}(33 \mathrm{C})$ & 10655 & 7249 & 5978 & 116 \\
\hline $\mathrm{H}(33 \mathrm{D})$ & 10064 & 8175 & 5689 & 120 \\
\hline $\mathrm{H}(33 \mathrm{E})$ & 9330 & 8095 & 4994 & 97 \\
\hline $\mathrm{H}(41 \mathrm{~A})$ & 7827 & 3415 & 4132 & 125 \\
\hline $\mathrm{H}(41 \mathrm{~B})$ & 8006 & 2572 & 4671 & 184 \\
\hline $\mathrm{H}(41 \mathrm{C})$ & 8086 & 2844 & 5414 & 182 \\
\hline
\end{tabular}




\begin{tabular}{|c|c|c|c|c|}
\hline $\mathrm{H}(41 \mathrm{D})$ & 7952 & 3996 & 5621 & 156 \\
\hline $\mathrm{H}(41 \mathrm{E})$ & 7754 & 4871 & 5062 & 105 \\
\hline $\mathrm{H}(42 \mathrm{~A})$ & 6637 & 4176 & 4328 & 101 \\
\hline $\mathrm{H}(42 \mathrm{~B})$ & 5617 & 4334 & 4252 & 114 \\
\hline $\mathrm{H}(42 \mathrm{C})$ & 5182 & 5403 & 4064 & 101 \\
\hline $\mathrm{H}(42 \mathrm{D})$ & 5725 & 6268 & 3838 & 103 \\
\hline $\mathrm{H}(42 \mathrm{E})$ & 6749 & 6098 & 3873 & 90 \\
\hline $\mathrm{H}(43 \mathrm{~A})$ & 8324 & 4032 & 3712 & 105 \\
\hline $\mathrm{H}(43 \mathrm{~B})$ & 8190 & 3517 & 3010 & 125 \\
\hline $\mathrm{H}(43 \mathrm{C})$ & 7394 & 3805 & 2417 & 132 \\
\hline $\mathrm{H}(43 \mathrm{D})$ & 6699 & 4638 & 2523 & 117 \\
\hline $\mathrm{H}(43 \mathrm{E})$ & 6772 & 5081 & 3256 & 87 \\
\hline $\mathrm{H}(51 \mathrm{~A})$ & 6265 & 9363 & 3302 & 169 \\
\hline $\mathrm{H}(51 \mathrm{~B})$ & 6543 & 8687 & 3934 & 201 \\
\hline $\mathrm{H}(51 \mathrm{C})$ & 6228 & 7561 & 3921 & 150 \\
\hline $\mathrm{H}(51 \mathrm{D})$ & 5531 & 7176 & 3280 & 258 \\
\hline $\mathrm{H}(51 \mathrm{E})$ & 5294 & 7853 & 2644 & 183 \\
\hline $\mathrm{H}(52 \mathrm{~A})$ & 5505 & 8131 & 1772 & 130 \\
\hline $\mathrm{H}(52 \mathrm{~B})$ & 4784 & 7413 & 1292 & 146 \\
\hline $\mathrm{H}(52 \mathrm{C})$ & 3778 & 7569 & 1236 & 129 \\
\hline $\mathrm{H}(52 \mathrm{D})$ & 3469 & 8385 & 1693 & 127 \\
\hline $\mathrm{H}(52 \mathrm{E})$ & 4193 & 9093 & 2175 & 107 \\
\hline $\mathrm{H}(53 \mathrm{~A})$ & 5179 & 9734 & 3213 & 117 \\
\hline $\mathrm{H}(53 \mathrm{~B})$ & 4522 & 10618 & 3352 & 151 \\
\hline $\mathrm{H}(53 \mathrm{C})$ & 4015 & 11335 & 2784 & 125 \\
\hline $\mathrm{H}(53 \mathrm{D})$ & 4125 & 11194 & 2075 & 123 \\
\hline $\mathrm{H}(53 \mathrm{E})$ & 4748 & 10324 & 1929 & 109 \\
\hline $\mathrm{H}(61 \mathrm{~A})$ & 6613 & 10361 & 947 & 124 \\
\hline $\mathrm{H}(61 \mathrm{~B})$ & 6418 & 9900 & 231 & 148 \\
\hline $\mathrm{H}(61 \mathrm{C})$ & 6806 & 8856 & 112 & 144 \\
\hline $\mathrm{H}(61 \mathrm{D})$ & 7402 & 8243 & 708 & 139 \\
\hline $\mathrm{H}(61 \mathrm{E})$ & 7618 & 8698 & 1443 & 110 \\
\hline $\mathrm{H}(62 \mathrm{~A})$ & 7733 & 8706 & 2422 & 161 \\
\hline $\mathrm{H}(62 \mathrm{~B})$ & 8677 & 8224 & 2725 & 211 \\
\hline $\mathrm{H}(62 \mathrm{C})$ & 9531 & 8824 & 2645 & 274 \\
\hline $\mathrm{H}(62 \mathrm{D})$ & 9402 & 9944 & 2352 & 372 \\
\hline $\mathrm{H}(62 \mathrm{E})$ & 8454 & 10311 & 1950 & 265 \\
\hline $\mathrm{H}(63 \mathrm{~A})$ & 7269 & 11104 & 2419 & 141 \\
\hline
\end{tabular}




\begin{tabular}{lrrrr}
$\mathrm{H}(63 \mathrm{~B})$ & 7586 & 12244 & 2438 & 182 \\
$\mathrm{H}(63 \mathrm{C})$ & 7984 & 12674 & 1877 & 253 \\
$\mathrm{H}(63 \mathrm{D})$ & 7941 & 11989 & 1254 & 450 \\
$\mathrm{H}(63 \mathrm{E})$ & 7624 & 10862 & 1242 & 282 \\
$\mathrm{H}(41 \mathrm{~F})$ & 12246 & 6007 & 1754 & 271 \\
$\mathrm{H}(41 \mathrm{G})$ & 12109 & 5954 & 1224 & 271 \\
$\mathrm{H}(42 \mathrm{~F})$ & 3841 & 6422 & 4313 & 155 \\
$\mathrm{H}(42 \mathrm{G})$ & 4227 & 7105 & 4325 & 155 \\
\hline
\end{tabular}


Table S6. Atomic coordinates ( $\times 10^{4}$ ) and equivalent isotropic displacement parameters $\left(\AA^{2} \times 10^{3}\right)$ for $\mathrm{OsCl}(\mathrm{CH}=\mathrm{CH}(p$-tolyl $))(\mathrm{CO})\left(\mathrm{PPh}_{3}\right) 32 \mathrm{CH}_{2} \mathrm{Cl}_{2}\left(\mathbf{8 b} 2 \mathrm{CH}_{2} \mathrm{Cl}_{2}\right)$.

\begin{tabular}{|c|c|c|c|c|}
\hline & $\mathrm{x}$ & $\mathrm{y}$ & $\mathrm{z}$ & $\mathrm{U}(\mathrm{eq})$ \\
\hline Os(1) & $8644(1)$ & $2608(1)$ & $1669(1)$ & $12(1)$ \\
\hline $\mathrm{Cl}(1)$ & $9842(1)$ & $3013(1)$ & 1741(1) & $16(1)$ \\
\hline $\mathrm{P}(1)$ & $9123(1)$ & 1920(1) & $1074(1)$ & $14(1)$ \\
\hline $\mathrm{P}(2)$ & $8293(1)$ & $3466(1)$ & $2107(1)$ & $13(1)$ \\
\hline $\mathrm{P}(3)$ & $8597(1)$ & $2029(1)$ & $2504(1)$ & $14(1)$ \\
\hline$C(10)$ & $7740(4)$ & $2363(3)$ & $1542(3)$ & $17(2)$ \\
\hline $\mathrm{O}(1)$ & $7180(3)$ & $2222(2)$ & $1432(2)$ & $22(1)$ \\
\hline $\mathrm{C}(1)$ & $8541(4)$ & $3079(3)$ & $960(3)$ & $16(2)$ \\
\hline $\mathrm{C}(2)$ & $8054(4)$ & $3057(3)$ & $570(3)$ & $16(2)$ \\
\hline$C(3)$ & $7959(4)$ & $3427(3)$ & $108(3)$ & $19(2)$ \\
\hline$C(4)$ & $7318(4)$ & $3430(3)$ & $-168(3)$ & $24(2)$ \\
\hline $\mathrm{C}(5)$ & $7211(4)$ & $3761(3)$ & $-617(3)$ & $27(2)$ \\
\hline$C(6)$ & $7727(5)$ & $4126(3)$ & $-802(3)$ & $25(2)$ \\
\hline$C(7)$ & 8364(4) & 4137(3) & $-518(3)$ & $28(2)$ \\
\hline $\mathrm{C}(8)$ & $8480(4)$ & $3798(3)$ & $-74(3)$ & $21(2)$ \\
\hline $\mathrm{C}(9)$ & $7594(4)$ & $4469(3)$ & $-1292(3)$ & $31(2)$ \\
\hline $\mathrm{C}(11)$ & $9741(3)$ & $2108(3)$ & $531(3)$ & $14(2)$ \\
\hline$C(12)$ & 10042(4) & 1677(3) & $232(3)$ & $20(2)$ \\
\hline$C(13)$ & 10496(4) & $1772(3)$ & $-194(3)$ & $26(2)$ \\
\hline $\mathrm{C}(14)$ & $10642(4)$ & 2299(3) & $-348(3)$ & $29(2)$ \\
\hline$C(15)$ & $10356(4)$ & $2742(3)$ & $-58(3)$ & $25(2)$ \\
\hline$C(16)$ & $9907(4)$ & $2642(3)$ & $380(3)$ & $21(2)$ \\
\hline $\mathrm{C}(21)$ & $8422(3)$ & $1568(3)$ & $695(3)$ & $13(2)$ \\
\hline $\mathrm{C}(22)$ & 7961(4) & 1231(3) & $988(3)$ & $18(2)$ \\
\hline $\mathrm{C}(23)$ & 7389(4) & 982(3) & $737(3)$ & $24(2)$ \\
\hline$C(24)$ & $7265(4)$ & 1073(3) & $189(3)$ & $26(2)$ \\
\hline$C(25)$ & $7716(4)$ & $1402(3)$ & $-103(3)$ & $29(2)$ \\
\hline$C(26)$ & $8306(4)$ & $1653(3)$ & $151(3)$ & $22(2)$ \\
\hline $\mathrm{C}(31)$ & $9656(3)$ & 1363(3) & 1372(3) & $12(2)$ \\
\hline $\mathrm{C}(32)$ & 10227(4) & $1526(3)$ & $1688(3)$ & $20(2)$ \\
\hline$C(33)$ & 10663(4) & $1133(3)$ & 1914(3) & $26(2)$ \\
\hline$C(34)$ & 10554(4) & $580(3)$ & $1827(3)$ & $25(2)$ \\
\hline$C(35)$ & 9998(4) & $417(3)$ & 1498(3) & $24(2)$ \\
\hline
\end{tabular}




\begin{tabular}{|c|c|c|c|c|}
\hline$C(36)$ & $9554(4)$ & $799(3)$ & $1267(3)$ & $23(2)$ \\
\hline $\mathrm{C}(41)$ & $8632(4)$ & $4079(3)$ & $1758(3)$ & $15(2)$ \\
\hline$C(42)$ & $8302(4)$ & $4290(3)$ & $1289(3)$ & $19(2)$ \\
\hline $\mathrm{C}(43)$ & $8584(4)$ & $4735(3)$ & 1013(3) & $24(2)$ \\
\hline$C(44)$ & $9190(4)$ & $4982(3)$ & 1197(3) & $28(2)$ \\
\hline$C(45)$ & $9524(4)$ & $4786(3)$ & $1656(3)$ & $26(2)$ \\
\hline$C(46)$ & $9253(4)$ & $4338(3)$ & 1927(3) & $21(2)$ \\
\hline $\mathrm{C}(51)$ & $8594(3)$ & $3633(3)$ & 2793(3) & 11(1) \\
\hline$C(52)$ & $8419(4)$ & $4135(3)$ & $3042(3)$ & $16(2)$ \\
\hline $\mathrm{C}(53)$ & $8677(4)$ & $4264(3)$ & $3550(3)$ & $20(2)$ \\
\hline$C(54)$ & 9111(4) & 3892(3) & $3824(3)$ & $19(2)$ \\
\hline $\mathrm{C}(55)$ & 9299(4) & $3399(3)$ & $3579(3)$ & $19(2)$ \\
\hline$C(56)$ & $9044(4)$ & $3279(3)$ & $3060(3)$ & $16(2)$ \\
\hline $\mathrm{C}(61)$ & $7337(3)$ & $3586(3)$ & $2120(3)$ & $14(2)$ \\
\hline$C(62)$ & $6945(4)$ & $3502(3)$ & 1643(3) & $20(2)$ \\
\hline $\mathrm{C}(63)$ & $6223(4)$ & $3581(3)$ & $1637(3)$ & $26(2)$ \\
\hline$C(64)$ & $5864(4)$ & $3738(3)$ & 2096(3) & $21(2)$ \\
\hline$C(65)$ & $6241(4)$ & $3819(3)$ & 2572(3) & $24(2)$ \\
\hline$C(66)$ & $6958(4)$ & $3741(3)$ & $2580(3)$ & $16(2)$ \\
\hline $\mathrm{C}(71)$ & $9366(4)$ & 1868(3) & 2927(3) & $15(2)$ \\
\hline$C(72)$ & $9325(4)$ & $1475(3)$ & $3342(3)$ & $17(2)$ \\
\hline$C(73)$ & 9914(4) & $1364(3)$ & $3664(3)$ & $23(2)$ \\
\hline$C(74)$ & $10546(4)$ & $1638(3)$ & $3565(3)$ & $24(2)$ \\
\hline$C(75)$ & 10593(4) & 2020(3) & $3160(3)$ & $23(2)$ \\
\hline$C(76)$ & 10012(4) & 2134(3) & 2841(3) & $18(2)$ \\
\hline $\mathrm{C}(81)$ & 7951(3) & $2286(3)$ & $3009(3)$ & $14(2)$ \\
\hline $\mathrm{C}(82)$ & $8103(4)$ & $2331(3)$ & $3553(3)$ & $20(2)$ \\
\hline $\mathrm{C}(83)$ & $7595(4)$ & $2496(3)$ & $3917(3)$ & $28(2)$ \\
\hline $\mathrm{C}(84)$ & $6910(4)$ & $2619(3)$ & $3751(3)$ & $28(2)$ \\
\hline$C(85)$ & $6758(4)$ & $2583(3)$ & $3210(3)$ & $23(2)$ \\
\hline$C(86)$ & $7269(4)$ & $2432(3)$ & 2838(3) & $16(2)$ \\
\hline $\mathrm{C}(91)$ & $8240(4)$ & $1329(3)$ & $2398(3)$ & $13(2)$ \\
\hline $\mathrm{C}(92)$ & $8697(4)$ & $871(3)$ & $2358(3)$ & $18(2)$ \\
\hline $\mathrm{C}(93)$ & $8438(4)$ & $346(3)$ & $2250(3)$ & $24(2)$ \\
\hline $\mathrm{C}(94)$ & $7722(4)$ & $262(3)$ & $2177(3)$ & $25(2)$ \\
\hline $\mathrm{C}(95)$ & $7259(4)$ & 701(3) & $2216(3)$ & $25(2)$ \\
\hline$C(96)$ & $7517(4)$ & 1219(3) & $2326(3)$ & $20(2)$ \\
\hline$C(1 S)$ & $6597(5)$ & $4915(4)$ & 131(3) & $37(2)$ \\
\hline
\end{tabular}




\begin{tabular}{lrrrr}
$\mathrm{Cl}(11)$ & $5874(1)$ & $4805(1)$ & $-297(1)$ & $47(1)$ \\
$\mathrm{Cl}(12)$ & $6489(1)$ & $4609(1)$ & $762(1)$ & $40(1)$ \\
$\mathrm{C}(2 \mathrm{~S})$ & $5635(5)$ & $1009(4)$ & $-778(3)$ & $36(2)$ \\
$\mathrm{Cl}(21)$ & $5342(1)$ & $672(1)$ & $-189(1)$ & $39(1)$ \\
$\mathrm{Cl}(22)$ & $6340(1)$ & $660(1)$ & $-1078(1)$ & $51(1)$ \\
\hline
\end{tabular}

$\mathrm{U}(\mathrm{eq})$ is defined as one third of the trace of the orthogonalized $\mathrm{U}^{\mathrm{ij}}$ tensor. 
Table S7. Bond lengths $[\AA]$ and angles $\left[{ }^{\circ}\right]$ for $\mathrm{OsCl}(\mathrm{CH}=\mathrm{CH}(p$ tolyl) $)(\mathrm{CO})\left(\mathrm{PPh}_{3}\right) 32 \mathrm{CH}_{2} \mathrm{Cl}_{2}\left(\mathbf{8 b} \cdot 2 \mathrm{CH}_{2} \mathrm{Cl}_{2}\right)$.

\begin{tabular}{|c|c|c|c|}
\hline Os(1)-C(10) & $1.837(7)$ & $\mathrm{Os}(1)-\mathrm{C}(1)$ & $2.105(7)$ \\
\hline Os(1)-P(1) & $2.4041(19)$ & $\operatorname{Os}(1)-\mathrm{P}(2)$ & $2.4386(19)$ \\
\hline Os(1)-Cl(1) & $2.4772(16)$ & Os(1)-P(3) & $2.5000(19)$ \\
\hline $\mathrm{P}(1)-\mathrm{C}(21)$ & $1.834(7)$ & $\mathrm{P}(1)-\mathrm{C}(31)$ & $1.840(7)$ \\
\hline $\mathrm{P}(1)-\mathrm{C}(11)$ & $1.840(7)$ & $\mathrm{P}(2)-\mathrm{C}(61)$ & $1.833(7)$ \\
\hline $\mathrm{P}(2)-\mathrm{C}(51)$ & $1.836(6)$ & $\mathrm{P}(2)-\mathrm{C}(41)$ & $1.836(7)$ \\
\hline $\mathrm{P}(3)-\mathrm{C}(71)$ & $1.835(7)$ & $\mathrm{P}(3)-\mathrm{C}(91)$ & $1.846(7)$ \\
\hline $\mathrm{P}(3)-\mathrm{C}(81)$ & $1.856(7)$ & $\mathrm{C}(10)-\mathrm{O}(1)$ & $1.146(8)$ \\
\hline $\mathrm{C}(1)-\mathrm{C}(2)$ & $1.336(10)$ & $\mathrm{C}(2)-\mathrm{C}(3)$ & $1.464(10)$ \\
\hline $\mathrm{C}(3)-\mathrm{C}(4)$ & $1.393(10)$ & $\mathrm{C}(3)-\mathrm{C}(8)$ & $1.408(10)$ \\
\hline$C(4)-C(5)$ & $1.385(10)$ & $C(5)-C(6)$ & $1.397(11)$ \\
\hline$C(6)-C(7)$ & $1.395(11)$ & $\mathrm{C}(6)-\mathrm{C}(9)$ & $1.493(10)$ \\
\hline$C(7)-C(8)$ & $1.390(10)$ & $\mathrm{C}(11)-\mathrm{C}(16)$ & $1.382(10)$ \\
\hline $\mathrm{C}(11)-\mathrm{C}(12)$ & $1.400(10)$ & $\mathrm{C}(12)-\mathrm{C}(13)$ & $1.379(10)$ \\
\hline $\mathrm{C}(13)-\mathrm{C}(14)$ & $1.361(11)$ & $\mathrm{C}(14)-\mathrm{C}(15)$ & $1.400(10)$ \\
\hline$C(15)-C(16)$ & $1.399(10)$ & $C(21)-C(26)$ & $1.380(10)$ \\
\hline $\mathrm{C}(21)-\mathrm{C}(22)$ & $1.398(10)$ & $C(22)-C(23)$ & $1.386(10)$ \\
\hline $\mathrm{C}(23)-\mathrm{C}(24)$ & $1.396(11)$ & $C(24)-C(25)$ & $1.373(11)$ \\
\hline$C(25)-C(26)$ & $1.418(10)$ & $\mathrm{C}(31)-\mathrm{C}(32)$ & 1.391(10) \\
\hline $\mathrm{C}(31)-\mathrm{C}(36)$ & $1.406(10)$ & $\mathrm{C}(32)-\mathrm{C}(33)$ & $1.380(10)$ \\
\hline $\mathrm{C}(33)-\mathrm{C}(34)$ & $1.373(10)$ & $\mathrm{C}(34)-\mathrm{C}(35)$ & $1.388(11)$ \\
\hline$C(35)-C(36)$ & $1.376(10)$ & $\mathrm{C}(41)-\mathrm{C}(46)$ & $1.397(10)$ \\
\hline $\mathrm{C}(41)-\mathrm{C}(42)$ & $1.415(10)$ & $\mathrm{C}(42)-\mathrm{C}(43)$ & $1.384(10)$ \\
\hline $\mathrm{C}(43)-\mathrm{C}(44)$ & $1.372(11)$ & $\mathrm{C}(44)-\mathrm{C}(45)$ & $1.386(11)$ \\
\hline $\mathrm{C}(45)-\mathrm{C}(46)$ & $1.374(10)$ & $C(51)-C(56)$ & $1.377(9)$ \\
\hline $\mathrm{C}(51)-\mathrm{C}(52)$ & $1.404(9)$ & $C(52)-C(53)$ & $1.387(10)$ \\
\hline $\mathrm{C}(53)-\mathrm{C}(54)$ & $1.394(10)$ & $\mathrm{C}(54)-\mathrm{C}(55)$ & $1.388(10)$ \\
\hline$C(55)-C(56)$ & $1.404(9)$ & $\mathrm{C}(61)-\mathrm{C}(66)$ & 1.398(9) \\
\hline $\mathrm{C}(61)-\mathrm{C}(62)$ & $1.409(10)$ & $\mathrm{C}(62)-\mathrm{C}(63)$ & $1.379(10)$ \\
\hline $\mathrm{C}(63)-\mathrm{C}(64)$ & $1.378(10)$ & $\mathrm{C}(64)-\mathrm{C}(65)$ & 1.391(10) \\
\hline $\mathrm{C}(65)-\mathrm{C}(66)$ & $1.369(9)$ & $\mathrm{C}(71)-\mathrm{C}(76)$ & $1.397(10)$ \\
\hline$C(71)-C(72)$ & $1.403(10)$ & $\mathrm{C}(72)-\mathrm{C}(73)$ & $1.396(10)$ \\
\hline $\mathrm{C}(73)-\mathrm{C}(74)$ & $1.388(10)$ & $\mathrm{C}(74)-\mathrm{C}(75)$ & $1.367(10)$ \\
\hline $\mathrm{C}(75)-\mathrm{C}(76)$ & $1.382(10)$ & $\mathrm{C}(81)-\mathrm{C}(82)$ & $1.382(10)$ \\
\hline $\mathrm{C}(81)-\mathrm{C}(86)$ & $1.403(9)$ & $\mathrm{C}(82)-\mathrm{C}(83)$ & $1.375(10)$ \\
\hline
\end{tabular}




\begin{tabular}{|c|c|c|c|}
\hline $\mathrm{C}(83)-\mathrm{C}(84)$ & $1.392(11)$ & $\mathrm{C}(84)-\mathrm{C}(85)$ & $1.374(11)$ \\
\hline $\mathrm{C}(85)-\mathrm{C}(86)$ & $1.386(9)$ & $C(91)-C(96)$ & $1.404(9)$ \\
\hline $\mathrm{C}(91)-\mathrm{C}(92)$ & $1.410(10)$ & $\mathrm{C}(92)-\mathrm{C}(93)$ & $1.391(10)$ \\
\hline $\mathrm{C}(93)-\mathrm{C}(94)$ & $1.382(10)$ & $C(94)-C(95)$ & $1.382(11)$ \\
\hline $\mathrm{C}(95)-\mathrm{C}(96)$ & $1.375(10)$ & $\mathrm{C}(1 \mathrm{~S})-\mathrm{Cl}(12)$ & $1.744(9)$ \\
\hline $\mathrm{C}(1 \mathrm{~S})-\mathrm{Cl}(11)$ & $1.749(9)$ & $\mathrm{C}(2 \mathrm{~S})-\mathrm{Cl}(22)$ & $1.746(9)$ \\
\hline $\mathrm{C}(2 \mathrm{~S})-\mathrm{Cl}(21)$ & $1.760(9)$ & & \\
\hline $\mathrm{C}(10)-\mathrm{Os}(1)-\mathrm{C}(1)$ & $86.9(3)$ & $\mathrm{C}(10)-\mathrm{Os}(1)-\mathrm{P}(1)$ & $91.3(2)$ \\
\hline $\mathrm{C}(1)-\mathrm{Os}(1)-\mathrm{P}(1)$ & $84.23(18)$ & $\mathrm{C}(10)-\mathrm{Os}(1)-\mathrm{P}(2)$ & $95.6(2)$ \\
\hline $\mathrm{C}(1)-\mathrm{Os}(1)-\mathrm{P}(2)$ & $83.28(18)$ & $\mathrm{P}(1)-\mathrm{Os}(1)-\mathrm{P}(2)$ & $165.36(7)$ \\
\hline $\mathrm{C}(10)-\mathrm{Os}(1)-\mathrm{Cl}(1)$ & $172.8(2)$ & $\mathrm{C}(1)-\mathrm{Os}(1)-\mathrm{Cl}(1)$ & $85.9(2)$ \\
\hline $\mathrm{P}(1)-\mathrm{Os}(1)-\mathrm{Cl}(1)$ & $88.53(6)$ & $\mathrm{P}(2)-\mathrm{Os}(1)-\mathrm{Cl}(1)$ & $83.00(6)$ \\
\hline $\mathrm{C}(10)-\mathrm{Os}(1)-\mathrm{P}(3)$ & $85.9(2)$ & $\mathrm{C}(1)-\mathrm{Os}(1)-\mathrm{P}(3)$ & 172.61(19) \\
\hline $\mathrm{P}(1)-\mathrm{Os}(1)-\mathrm{P}(3)$ & $97.59(6)$ & $\mathrm{P}(2)-\mathrm{Os}(1)-\mathrm{P}(3)$ & $95.77(6)$ \\
\hline $\mathrm{Cl}(1)-\mathrm{Os}(1)-\mathrm{P}(3)$ & 101.30(6) & $\mathrm{C}(21)-\mathrm{P}(1)-\mathrm{C}(31)$ & $105.2(3)$ \\
\hline $\mathrm{C}(21)-\mathrm{P}(1)-\mathrm{C}(11)$ & $101.6(3)$ & $\mathrm{C}(31)-\mathrm{P}(1)-\mathrm{C}(11)$ & $97.3(3)$ \\
\hline $\mathrm{C}(21)-\mathrm{P}(1)-\mathrm{Os}(1)$ & $111.3(2)$ & $\mathrm{C}(31)-\mathrm{P}(1)-\mathrm{Os}(1)$ & $117.9(2)$ \\
\hline $\mathrm{C}(11)-\mathrm{P}(1)-\mathrm{Os}(1)$ & $121.0(2)$ & $\mathrm{C}(61)-\mathrm{P}(2)-\mathrm{C}(51)$ & $104.8(3)$ \\
\hline $\mathrm{C}(61)-\mathrm{P}(2)-\mathrm{C}(41)$ & 103.0(3) & $\mathrm{C}(51)-\mathrm{P}(2)-\mathrm{C}(41)$ & $98.6(3)$ \\
\hline $\mathrm{C}(61)-\mathrm{P}(2)-\mathrm{Os}(1)$ & $114.3(2)$ & $\mathrm{C}(51)-\mathrm{P}(2)-\mathrm{Os}(1)$ & $121.0(2)$ \\
\hline $\mathrm{C}(41)-\mathrm{P}(2)-\mathrm{Os}(1)$ & $112.7(2)$ & $\mathrm{C}(71)-\mathrm{P}(3)-\mathrm{C}(91)$ & $100.1(3)$ \\
\hline $\mathrm{C}(71)-\mathrm{P}(3)-\mathrm{C}(81)$ & $102.1(3)$ & $\mathrm{C}(91)-\mathrm{P}(3)-\mathrm{C}(81)$ & $99.4(3)$ \\
\hline $\mathrm{C}(71)-\mathrm{P}(3)-\mathrm{Os}(1)$ & $124.3(2)$ & C(91)-P(3)-Os(1) & $114.3(2)$ \\
\hline $\mathrm{C}(81)-\mathrm{P}(3)-\mathrm{Os}(1)$ & $113.2(2)$ & $\mathrm{O}(1)-\mathrm{C}(10)-\mathrm{Os}(1)$ & $176.0(6)$ \\
\hline $\mathrm{C}(2)-\mathrm{C}(1)-\mathrm{Os}(1)$ & $130.1(6)$ & $\mathrm{C}(1)-\mathrm{C}(2)-\mathrm{C}(3)$ & $128.6(7)$ \\
\hline $\mathrm{C}(4)-\mathrm{C}(3)-\mathrm{C}(8)$ & 116.6(7) & $\mathrm{C}(4)-\mathrm{C}(3)-\mathrm{C}(2)$ & $119.6(7)$ \\
\hline $\mathrm{C}(8)-\mathrm{C}(3)-\mathrm{C}(2)$ & $123.8(7)$ & $C(5)-C(4)-C(3)$ & $121.6(8)$ \\
\hline$C(4)-C(5)-C(6)$ & $121.9(7)$ & $C(7)-C(6)-C(5)$ & $116.8(7)$ \\
\hline$C(7)-C(6)-C(9)$ & $123.1(8)$ & $C(5)-C(6)-C(9)$ & 120.1(8) \\
\hline$C(8)-C(7)-C(6)$ & $121.6(8)$ & $\mathrm{C}(7)-\mathrm{C}(8)-\mathrm{C}(3)$ & $121.4(7)$ \\
\hline$C(16)-C(11)-C(12)$ & $117.7(6)$ & $\mathrm{C}(16)-\mathrm{C}(11)-\mathrm{P}(1)$ & $125.0(5)$ \\
\hline $\mathrm{C}(12)-\mathrm{C}(11)-\mathrm{P}(1)$ & $117.3(6)$ & $\mathrm{C}(13)-\mathrm{C}(12)-\mathrm{C}(11)$ & $122.2(7)$ \\
\hline$C(14)-C(13)-C(12)$ & 119.7(8) & $\mathrm{C}(13)-\mathrm{C}(14)-\mathrm{C}(15)$ & 119.9(8) \\
\hline$C(16)-C(15)-C(14)$ & $120.0(7)$ & $\mathrm{C}(11)-\mathrm{C}(16)-\mathrm{C}(15)$ & $120.5(7)$ \\
\hline$C(26)-C(21)-C(22)$ & 119.7(7) & $\mathrm{C}(26)-\mathrm{C}(21)-\mathrm{P}(1)$ & $123.0(6)$ \\
\hline $\mathrm{C}(22)-\mathrm{C}(21)-\mathrm{P}(1)$ & $117.2(6)$ & $\mathrm{C}(23)-\mathrm{C}(22)-\mathrm{C}(21)$ & $120.6(7)$ \\
\hline$C(22)-C(23)-C(24)$ & $119.8(8)$ & $\mathrm{C}(25)-\mathrm{C}(24)-\mathrm{C}(23)$ & $120.0(8)$ \\
\hline
\end{tabular}




$\begin{array}{llll}\mathrm{C}(24)-\mathrm{C}(25)-\mathrm{C}(26) & 120.3(8) & \mathrm{C}(21)-\mathrm{C}(26)-\mathrm{C}(25) & 119.6(7) \\ \mathrm{C}(32)-\mathrm{C}(31)-\mathrm{C}(36) & 119.2(7) & \mathrm{C}(32)-\mathrm{C}(31)-\mathrm{P}(1) & 116.3(5) \\ \mathrm{C}(36)-\mathrm{C}(31)-\mathrm{P}(1) & 124.3(6) & \mathrm{C}(33)-\mathrm{C}(32)-\mathrm{C}(31) & 119.7(7) \\ \mathrm{C}(34)-\mathrm{C}(33)-\mathrm{C}(32) & 121.3(8) & \mathrm{C}(33)-\mathrm{C}(34)-\mathrm{C}(35) & 119.0(7) \\ \mathrm{C}(36)-\mathrm{C}(35)-\mathrm{C}(34) & 121.0(7) & \mathrm{C}(35)-\mathrm{C}(36)-\mathrm{C}(31) & 119.6(7) \\ \mathrm{C}(46)-\mathrm{C}(41)-\mathrm{C}(42) & 117.1(6) & \mathrm{C}(46)-\mathrm{C}(41)-\mathrm{P}(2) & 121.1(5) \\ \mathrm{C}(42)-\mathrm{C}(41)-\mathrm{P}(2) & 121.7(6) & \mathrm{C}(43)-\mathrm{C}(42)-\mathrm{C}(41) & 121.1(7) \\ \mathrm{C}(44)-\mathrm{C}(43)-\mathrm{C}(42) & 119.9(7) & \mathrm{C}(43)-\mathrm{C}(44)-\mathrm{C}(45) & 120.2(7) \\ \mathrm{C}(46)-\mathrm{C}(45)-\mathrm{C}(44) & 120.2(7) & \mathrm{C}(45)-\mathrm{C}(46)-\mathrm{C}(41) & 121.4(7) \\ \mathrm{C}(56)-\mathrm{C}(51)-\mathrm{C}(52) & 118.4(6) & \mathrm{C}(56)-\mathrm{C}(51)-\mathrm{P}(2) & 119.9(5) \\ \mathrm{C}(52)-\mathrm{C}(51)-\mathrm{P}(2) & 121.6(5) & \mathrm{C}(53)-\mathrm{C}(52)-\mathrm{C}(51) & 120.7(7) \\ \mathrm{C}(52)-\mathrm{C}(53)-\mathrm{C}(54) & 120.2(7) & \mathrm{C}(55)-\mathrm{C}(54)-\mathrm{C}(53) & 119.7(7) \\ \mathrm{C}(54)-\mathrm{C}(55)-\mathrm{C}(56) & 119.4(7) & \mathrm{C}(51)-\mathrm{C}(56)-\mathrm{C}(55) & 121.5(7) \\ \mathrm{C}(66)-\mathrm{C}(61)-\mathrm{C}(62) & 116.8(6) & \mathrm{C}(66)-\mathrm{C}(61)-\mathrm{P}(2) & 124.3(5) \\ \mathrm{C}(62)-\mathrm{C}(61)-\mathrm{P}(2) & 118.8(5) & \mathrm{C}(63)-\mathrm{C}(62)-\mathrm{C}(61) & 120.7(7) \\ \mathrm{C}(64)-\mathrm{C}(63)-\mathrm{C}(62) & 121.1(7) & \mathrm{C}(63)-\mathrm{C}(64)-\mathrm{C}(65) & 119.1(7) \\ \mathrm{C}(66)-\mathrm{C}(65)-\mathrm{C}(64) & 120.0(7) & \mathrm{C}(65)-\mathrm{C}(66)-\mathrm{C}(61) & 122.3(7) \\ \mathrm{C}(76)-\mathrm{C}(71)-\mathrm{C}(72) & 118.2(6) & \mathrm{C}(76)-\mathrm{C}(71)-\mathrm{P}(3) & 120.6(5) \\ \mathrm{C}(72)-\mathrm{C}(71)-\mathrm{P}(3) & 121.2(5) & \mathrm{C}(73)-\mathrm{C}(72)-\mathrm{C}(71) & 120.3(7) \\ \mathrm{C}(74)-\mathrm{C}(73)-\mathrm{C}(72) & 119.6(7) & \mathrm{C}(75)-\mathrm{C}(74)-\mathrm{C}(73) & 120.7(7) \\ \mathrm{C}(74)-\mathrm{C}(75)-\mathrm{C}(76) & 120.1(7) & \mathrm{C}(75)-\mathrm{C}(76)-\mathrm{C}(71) & 121.1(7) \\ \mathrm{C}(82)-\mathrm{C}(81)-\mathrm{C}(86) & 117.8(7) & \mathrm{C}(82)-\mathrm{C}(81)-\mathrm{P}(3) & 123.2(5) \\ \mathrm{C}(86)-\mathrm{C}(81)-\mathrm{P}(3) & 119.0(6) & \mathrm{C}(83)-\mathrm{C}(82)-\mathrm{C}(81) & 121.1(7) \\ \mathrm{C}(82)-\mathrm{C}(83)-\mathrm{C}(84) & 121.3(8) & \mathrm{C}(85)-\mathrm{C}(84)-\mathrm{C}(83) & 117.9(7) \\ \mathrm{C}(84)-\mathrm{C}(85)-\mathrm{C}(86) & 121.4(7) & \mathrm{C}(85)-\mathrm{C}(86)-\mathrm{C}(81) & 120.4(7) \\ \mathrm{C}(96)-\mathrm{C}(91)-\mathrm{C}(92) & 116.1(7) & \mathrm{C}(96)-\mathrm{C}(91)-\mathrm{P}(3) & 123.2(6) \\ \mathrm{C}(92)-\mathrm{C}(91)-\mathrm{P}(3) & 120.6(5) & \mathrm{C}(93)-\mathrm{C}(92)-\mathrm{C}(91) & 121.1(7) \\ \mathrm{C}(94)-\mathrm{C}(93)-\mathrm{C}(92) & 120.4(7) & \mathrm{C}(93)-\mathrm{C}(94)-\mathrm{C}(95) & 120.0(7) \\ \mathrm{C}(96)-\mathrm{C}(95)-\mathrm{C}(94) & 119.4(7) & \mathrm{C}(95)-\mathrm{C}(96)-\mathrm{C}(91) & 123.0(7) \\ \mathrm{Cl}(12)-\mathrm{C}(1 \mathrm{~S})-\mathrm{Cl}(11) & 112.8(5) & \mathrm{Cl}(22)-\mathrm{C}(2 \mathrm{~S})-\mathrm{Cl}(21) & 111.6(5)\end{array}$


Table S8. Anisotropic displacement parameters $\left(\AA^{2} \times 10^{3}\right)$ for $\mathrm{OsCl}(\mathrm{CH}=\mathrm{CH}(p$ tolyl))(CO)(PPh3) $32 \mathrm{CH}_{2} \mathrm{Cl}_{2}\left(\mathbf{8 b} \cdot 2 \mathrm{CH}_{2} \mathrm{Cl}_{2}\right)$.

\begin{tabular}{|c|c|c|c|c|c|c|}
\hline & $\mathrm{U}^{11}$ & $\mathrm{U}^{22}$ & $\mathrm{U}^{33}$ & $\mathrm{U}^{23}$ & $\mathrm{U}^{13}$ & $\mathrm{U}^{12}$ \\
\hline- & & & & & & \\
\hline Os(1) & $12(1)$ & $11(1)$ & $12(1)$ & $0(1)$ & $0(1)$ & $0(1)$ \\
\hline $\mathrm{Cl}(1)$ & $16(1)$ & $16(1)$ & $18(1)$ & $0(1)$ & $0(1)$ & $-2(1)$ \\
\hline $\mathrm{P}(1)$ & $12(1)$ & $16(1)$ & $15(1)$ & $-1(1)$ & $1(1)$ & $-1(1)$ \\
\hline $\mathrm{P}(2)$ & $14(1)$ & $14(1)$ & $13(1)$ & $-1(1)$ & $-2(1)$ & 1(1) \\
\hline $\mathrm{P}(3)$ & $12(1)$ & $14(1)$ & $17(1)$ & $0(1)$ & $0(1)$ & $-1(1)$ \\
\hline$C(10)$ & $15(3)$ & $25(4)$ & $11(4)$ & $3(3)$ & $-3(3)$ & $-3(3)$ \\
\hline $\mathrm{O}(1)$ & $23(3)$ & $20(3)$ & $22(3)$ & $-3(2)$ & $6(2)$ & $-4(2)$ \\
\hline $\mathrm{C}(1)$ & $19(4)$ & $12(4)$ & $16(4)$ & $-6(3)$ & $12(3)$ & 1(3) \\
\hline$C(2)$ & $14(3)$ & $16(4)$ & $16(4)$ & $-2(3)$ & $8(3)$ & $-3(3)$ \\
\hline$C(3)$ & $26(4)$ & $26(5)$ & $7(4)$ & $-5(3)$ & $0(3)$ & $13(3)$ \\
\hline $\mathrm{C}(4)$ & $29(4)$ & $25(5)$ & $18(5)$ & $-1(4)$ & $-11(4)$ & $4(4)$ \\
\hline$C(5)$ & $27(4)$ & $28(5)$ & $25(5)$ & $-1(4)$ & $-11(4)$ & $6(4)$ \\
\hline$C(6)$ & $48(5)$ & $18(5)$ & $9(4)$ & $4(3)$ & $3(4)$ & $9(4)$ \\
\hline$C(7)$ & $36(5)$ & $22(5)$ & $26(5)$ & $7(4)$ & $6(4)$ & $5(4)$ \\
\hline $\mathrm{C}(8)$ & $18(4)$ & $26(5)$ & $17(4)$ & $3(4)$ & $-6(3)$ & $0(3)$ \\
\hline $\mathrm{C}(9)$ & $44(5)$ & $31(5)$ & $19(5)$ & $-3(4)$ & $0(4)$ & $7(4)$ \\
\hline $\mathrm{C}(11)$ & $17(4)$ & $17(4)$ & $7(4)$ & $3(3)$ & $0(3)$ & $0(3)$ \\
\hline $\mathrm{C}(12)$ & $27(4)$ & $17(4)$ & $17(4)$ & $1(3)$ & $4(3)$ & $3(3)$ \\
\hline$C(13)$ & $28(4)$ & $21(5)$ & $29(5)$ & $-3(4)$ & 1(4) & $4(4)$ \\
\hline$C(14)$ & $35(5)$ & $35(6)$ & $18(5)$ & $-2(4)$ & $3(4)$ & $5(4)$ \\
\hline$C(15)$ & $28(4)$ & $17(5)$ & $31(5)$ & $0(4)$ & $7(4)$ & $-4(3)$ \\
\hline$C(16)$ & $28(4)$ & $20(4)$ & $16(4)$ & $-8(4)$ & $6(3)$ & $2(4)$ \\
\hline$C(21)$ & $15(3)$ & $4(4)$ & $19(4)$ & $-6(3)$ & $-1(3)$ & $0(3)$ \\
\hline$C(22)$ & $15(4)$ & $23(5)$ & $16(4)$ & $1(3)$ & $6(3)$ & $4(3)$ \\
\hline $\mathrm{C}(23)$ & $22(4)$ & $19(5)$ & $31(5)$ & $-7(4)$ & $2(4)$ & $-2(3)$ \\
\hline $\mathrm{C}(24)$ & $24(4)$ & $9(4)$ & $45(6)$ & $2(4)$ & $-6(4)$ & $3(3)$ \\
\hline$C(25)$ & $39(5)$ & $28(5)$ & $19(5)$ & $6(4)$ & $-18(4)$ & $-4(4)$ \\
\hline$C(26)$ & $28(4)$ & $18(5)$ & $20(5)$ & $4(3)$ & $0(3)$ & $-4(3)$ \\
\hline $\mathrm{C}(31)$ & $14(3)$ & $12(4)$ & $10(4)$ & $-1(3)$ & $7(3)$ & $3(3)$ \\
\hline$C(32)$ & $30(4)$ & $9(4)$ & $22(4)$ & $-2(4)$ & $2(4)$ & $0(3)$ \\
\hline$C(33)$ & $30(4)$ & $27(5)$ & $20(4)$ & $-1(4)$ & $-4(4)$ & $4(4)$ \\
\hline $\mathrm{C}(34)$ & $41(5)$ & $19(5)$ & $16(4)$ & 1(3) & $0(4)$ & $16(4)$ \\
\hline
\end{tabular}




\begin{tabular}{|c|c|c|c|c|c|c|}
\hline$C(35)$ & 29(4) & $17(5)$ & $25(5)$ & $-1(4)$ & $4(4)$ & $5(3)$ \\
\hline$C(36)$ & 29(4) & $22(5)$ & 19(4) & $-5(4)$ & $4(4)$ & $-3(3)$ \\
\hline $\mathrm{C}(41)$ & 23(3) & $11(4)$ & $12(4)$ & $-1(3)$ & $6(3)$ & $0(3)$ \\
\hline$C(42)$ & $27(4)$ & $13(4)$ & 16(4) & $-2(3)$ & $-3(3)$ & $3(3)$ \\
\hline $\mathrm{C}(43)$ & $40(5)$ & $16(4)$ & $17(4)$ & $2(3)$ & $-1(4)$ & $8(4)$ \\
\hline $\mathrm{C}(44)$ & $47(5)$ & $16(5)$ & $22(5)$ & 1(4) & $14(4)$ & $0(4)$ \\
\hline $\mathrm{C}(45)$ & 29(4) & $19(4)$ & $28(5)$ & $-9(4)$ & $-3(4)$ & $-7(3)$ \\
\hline$C(46)$ & $15(4)$ & $19(4)$ & $29(5)$ & $6(4)$ & $0(3)$ & $-1(3)$ \\
\hline $\mathrm{C}(51)$ & 10(3) & $17(4)$ & $4(3)$ & $-4(3)$ & $-3(3)$ & $-1(3)$ \\
\hline $\mathrm{C}(52)$ & $16(4)$ & $17(4)$ & 16(4) & $2(3)$ & $0(3)$ & $2(3)$ \\
\hline$C(53)$ & 21(4) & $17(4)$ & 22(4) & $-10(3)$ & $-1(4)$ & $-2(3)$ \\
\hline $\mathrm{C}(54)$ & $18(4)$ & $27(5)$ & 11(4) & $5(3)$ & $-3(3)$ & $-4(3)$ \\
\hline $\mathrm{C}(55)$ & $18(4)$ & $26(5)$ & $13(4)$ & $5(3)$ & $-7(3)$ & $-9(3)$ \\
\hline$C(56)$ & 17(4) & $15(4)$ & 16(4) & $3(3)$ & $4(3)$ & $-9(3)$ \\
\hline$C(61)$ & 12(3) & $14(4)$ & $17(4)$ & $5(3)$ & $0(3)$ & $5(3)$ \\
\hline $\mathrm{C}(62)$ & 23(4) & $12(4)$ & $24(4)$ & $-5(4)$ & $6(4)$ & 1(3) \\
\hline$C(63)$ & $24(4)$ & $28(5)$ & $27(5)$ & $-9(4)$ & $-8(4)$ & $-3(3)$ \\
\hline$C(64)$ & $16(4)$ & $25(5)$ & 21(5) & $-7(4)$ & $-7(3)$ & $-2(3)$ \\
\hline$C(65)$ & $24(5)$ & $23(5)$ & $25(5)$ & $-4(4)$ & $9(4)$ & $-3(3)$ \\
\hline$C(66)$ & $18(4)$ & $9(4)$ & 22(4) & 1(3) & $-1(3)$ & 1(3) \\
\hline$C(71)$ & $13(3)$ & $18(4)$ & $15(4)$ & $1(3)$ & $-1(3)$ & $4(3)$ \\
\hline $\mathrm{C}(72)$ & 18(3) & $19(4)$ & 14(4) & $-5(4)$ & $0(3)$ & $5(3)$ \\
\hline $\mathrm{C}(73)$ & $32(4)$ & $14(4)$ & $23(5)$ & $0(4)$ & $0(4)$ & $6(3)$ \\
\hline $\mathrm{C}(74)$ & $25(4)$ & $24(5)$ & $23(5)$ & $-4(4)$ & $-8(4)$ & $2(4)$ \\
\hline$C(75)$ & $9(3)$ & $31(5)$ & $27(5)$ & $-1(4)$ & $-2(3)$ & $-1(3)$ \\
\hline$C(76)$ & $26(4)$ & $18(4)$ & $10(4)$ & $4(3)$ & $3(3)$ & $3(3)$ \\
\hline $\mathrm{C}(81)$ & 17(4) & $7(4)$ & 20(4) & $0(3)$ & $7(3)$ & $1(3)$ \\
\hline $\mathrm{C}(82)$ & $26(4)$ & $20(5)$ & 14(4) & $-2(4)$ & $2(3)$ & $-4(4)$ \\
\hline $\mathrm{C}(83)$ & $42(5)$ & $20(5)$ & 22(4) & $-4(3)$ & $7(4)$ & $-5(4)$ \\
\hline$C(84)$ & $32(4)$ & $20(4)$ & $32(5)$ & $-2(4)$ & $20(4)$ & $3(4)$ \\
\hline$C(85)$ & 19(4) & $23(4)$ & $26(5)$ & $1(4)$ & $9(3)$ & $-1(3)$ \\
\hline$C(86)$ & 22(4) & $15(4)$ & $12(4)$ & $6(3)$ & $2(3)$ & $3(3)$ \\
\hline $\mathrm{C}(91)$ & 21(4) & $12(4)$ & $7(4)$ & $3(3)$ & $4(3)$ & $-5(3)$ \\
\hline $\mathrm{C}(92)$ & 23(4) & 19(4) & 12(4) & $6(3)$ & $9(3)$ & $1(3)$ \\
\hline C(93) & $45(5)$ & $11(4)$ & 17(4) & $1(3)$ & $6(4)$ & $4(3)$ \\
\hline $\mathrm{C}(94)$ & $41(5)$ & $16(5)$ & $18(5)$ & $1(3)$ & $4(4)$ & $-14(4)$ \\
\hline $\mathrm{C}(95)$ & 21(4) & $30(5)$ & $23(5)$ & $-3(4)$ & $1(3)$ & $-11(4)$ \\
\hline $\mathrm{C}(96)$ & 22(4) & $17(4)$ & $20(5)$ & $0(3)$ & $2(3)$ & $-1(3)$ \\
\hline
\end{tabular}




$\begin{array}{lllllll}\mathrm{C}(1 \mathrm{~S}) & 56(6) & 27(5) & 28(5) & -7(4) & 1(4) & -10(4) \\ \mathrm{Cl}(11) & 50(1) & 49(2) & 43(2) & 15(1) & -17(1) & -11(1) \\ \mathrm{Cl}(12) & 55(2) & 35(1) & 31(1) & 0(1) & -11(1) & 12(1) \\ \mathrm{C}(2 \mathrm{~S}) & 48(6) & 32(6) & 29(5) & -4(4) & 2(4) & 5(4) \\ \mathrm{Cl}(21) & 30(1) & 48(2) & 39(1) & 4(1) & 0(1) & -2(1) \\ \mathrm{Cl}(22) & 58(2) & 67(2) & 28(1) & 4(1) & 2(1) & 24(2)\end{array}$

The anisotropic displacement factor exponent takes the form: $-2 \pi^{2}\left[h^{2} a^{* 2} U^{11}+\ldots+2 h k a^{*} b^{*} U^{12}\right]$. 
Table S9. Hydrogen coordinates $\left(\times 10^{4}\right)$ and isotropic displacement parameters $\left(\AA^{2} \times 10^{3}\right)$ for $\mathrm{OsCl}(\mathrm{CH}=\mathrm{CH}(p$-tolyl $))(\mathrm{CO})\left(\mathrm{PPh}_{3}\right) 3 \cdot 2 \mathrm{CH}_{2} \mathrm{Cl}_{2}\left(\mathbf{8 b} \cdot 2 \mathrm{CH}_{2} \mathrm{Cl}_{2}\right)$.

\begin{tabular}{|c|c|c|c|c|}
\hline & $\mathrm{x}$ & $\mathrm{y}$ & $\mathrm{z}$ & $\mathrm{U}(\mathrm{eq})$ \\
\hline $\mathrm{H}(1 \mathrm{~A})$ & 8898 & 3350 & 909 & 19 \\
\hline $\mathrm{H}(2 \mathrm{~A})$ & 7725 & 2762 & 596 & 19 \\
\hline $\mathrm{H}(4 \mathrm{~A})$ & 6944 & 3199 & -46 & 29 \\
\hline $\mathrm{H}(5 \mathrm{~A})$ & 6773 & 3739 & -804 & 32 \\
\hline $\mathrm{H}(7 \mathrm{~A})$ & 8727 & 4382 & -631 & 34 \\
\hline $\mathrm{H}(8 \mathrm{~A})$ & 8919 & 3817 & 110 & 25 \\
\hline $\mathrm{H}(9 \mathrm{~A})$ & 7115 & 4398 & -1425 & 47 \\
\hline $\mathrm{H}(9 \mathrm{~B})$ & 7937 & 4372 & -1573 & 47 \\
\hline $\mathrm{H}(9 \mathrm{C})$ & 7642 & 4860 & -1201 & 47 \\
\hline $\mathrm{H}(12 \mathrm{~A})$ & 9931 & 1308 & 327 & 24 \\
\hline $\mathrm{H}(13 \mathrm{~A})$ & 10706 & 1471 & -379 & 31 \\
\hline $\mathrm{H}(14 \mathrm{~A})$ & 10936 & 2366 & -651 & 35 \\
\hline $\mathrm{H}(15 \mathrm{~A})$ & 10467 & 3110 & -158 & 30 \\
\hline $\mathrm{H}(16 \mathrm{~A})$ & 9713 & 2944 & 576 & 26 \\
\hline $\mathrm{H}(22 \mathrm{~A})$ & 8042 & 1171 & 1362 & 22 \\
\hline $\mathrm{H}(23 \mathrm{~A})$ & 7082 & 749 & 938 & 29 \\
\hline $\mathrm{H}(24 \mathrm{~A})$ & 6870 & 908 & 17 & 31 \\
\hline $\mathrm{H}(25 \mathrm{~A})$ & 7632 & 1463 & -476 & 34 \\
\hline $\mathrm{H}(26 \mathrm{~A})$ & 8620 & 1878 & -53 & 26 \\
\hline $\mathrm{H}(32 \mathrm{~A})$ & 10317 & 1907 & 1747 & 24 \\
\hline $\mathrm{H}(33 \mathrm{~A})$ & 11046 & 1247 & 2135 & 31 \\
\hline $\mathrm{H}(34 \mathrm{~A})$ & 10855 & 313 & 1988 & 30 \\
\hline $\mathrm{H}(35 \mathrm{~A})$ & 9922 & 35 & 1432 & 28 \\
\hline $\mathrm{H}(36 \mathrm{~A})$ & 9180 & 682 & 1037 & 28 \\
\hline $\mathrm{H}(42 \mathrm{~A})$ & 7879 & 4123 & 1161 & 23 \\
\hline $\mathrm{H}(43 \mathrm{~A})$ & 8358 & 4869 & 697 & 29 \\
\hline $\mathrm{H}(44 \mathrm{~A})$ & 9382 & 5289 & 1008 & 34 \\
\hline $\mathrm{H}(45 \mathrm{~A})$ & 9940 & 4961 & 1785 & 31 \\
\hline $\mathrm{H}(46 \mathrm{~A})$ & 9494 & 4202 & 2237 & 25 \\
\hline $\mathrm{H}(52 \mathrm{~A})$ & 8120 & 4389 & 2860 & 20 \\
\hline $\mathrm{H}(53 \mathrm{~A})$ & 8559 & 4607 & 3713 & 24 \\
\hline $\mathrm{H}(54 \mathrm{~A})$ & 9276 & 3976 & 4177 & 22 \\
\hline
\end{tabular}




\begin{tabular}{|c|c|c|c|c|}
\hline $\mathrm{H}(55 \mathrm{~A})$ & 9598 & 3145 & 3761 & 23 \\
\hline $\mathrm{H}(56 \mathrm{~A})$ & 9186 & 2946 & 2888 & 19 \\
\hline $\mathrm{H}(62 \mathrm{~A})$ & 7180 & 3390 & 1323 & 23 \\
\hline $\mathrm{H}(63 \mathrm{~A})$ & 5969 & 3527 & 1310 & 31 \\
\hline $\mathrm{H}(64 \mathrm{~A})$ & 5367 & 3789 & 2088 & 25 \\
\hline $\mathrm{H}(65 \mathrm{~A})$ & 6001 & 3929 & 2892 & 29 \\
\hline $\mathrm{H}(66 \mathrm{~A})$ & 7206 & 3795 & 2909 & 20 \\
\hline $\mathrm{H}(72 \mathrm{~A})$ & 8895 & 1283 & 3404 & 20 \\
\hline $\mathrm{H}(73 \mathrm{~A})$ & 9883 & 1103 & 3949 & 28 \\
\hline $\mathrm{H}(74 \mathrm{~A})$ & 10949 & 1558 & 3780 & 29 \\
\hline $\mathrm{H}(75 \mathrm{~A})$ & 11026 & 2208 & 3098 & 27 \\
\hline $\mathrm{H}(76 \mathrm{~A})$ & 10052 & 2398 & 2559 & 21 \\
\hline $\mathrm{H}(82 \mathrm{~A})$ & 8565 & 2247 & 3678 & 24 \\
\hline $\mathrm{H}(83 \mathrm{~A})$ & 7715 & 2527 & 4288 & 34 \\
\hline $\mathrm{H}(84 \mathrm{~A})$ & 6558 & 2725 & 4005 & 34 \\
\hline $\mathrm{H}(85 \mathrm{~A})$ & 6293 & 2663 & 3089 & 27 \\
\hline $\mathrm{H}(86 \mathrm{~A})$ & 7158 & 2427 & 2464 & 20 \\
\hline $\mathrm{H}(92 \mathrm{~A})$ & 9191 & 923 & 2406 & 22 \\
\hline $\mathrm{H}(93 \mathrm{~A})$ & 8755 & 43 & 2227 & 29 \\
\hline $\mathrm{H}(94 \mathrm{~A})$ & 7548 & -97 & 2099 & 30 \\
\hline $\mathrm{H}(95 \mathrm{~A})$ & 6766 & 645 & 2169 & 30 \\
\hline $\mathrm{H}(96 \mathrm{~A})$ & 7192 & 1517 & 2354 & 24 \\
\hline $\mathrm{H}(1 \mathrm{SA})$ & 7027 & 4765 & -42 & 45 \\
\hline $\mathrm{H}(1 \mathrm{SB})$ & 6666 & 5317 & 179 & 45 \\
\hline $\mathrm{H}(2 \mathrm{SA})$ & 5239 & 1034 & -1038 & 44 \\
\hline $\mathrm{H}(2 \mathrm{SB})$ & 5784 & 1388 & -686 & 44 \\
\hline
\end{tabular}

